\title{
An Immune Gene Expression Signature Associated With Development of Human Hepatocellular Carcinoma Identifies Mice That Respond to Chemopreventive Agents
}

\author{
Agrin Moeini,, Sara Torrecilla, ${ }^{1}$ Victoria Tovar, ${ }^{1}$ Carla Montironi, ${ }^{1,2}$ Carmen Andreu-Oller, ${ }^{1}$ \\ Judit Peix, ${ }^{1}$ Mónica Higuera, ${ }^{1,3}$ Dominik Pfister, ${ }^{4}$ Pierluigi Ramadori, ${ }^{4}$ Roser Pinyol, ${ }^{1}$ \\ Manel Solé, ${ }^{1}$ Mathias Heikenwälder, ${ }^{4}$ Scott L. Friedman, ${ }^{2}$ Daniela Sia, ${ }^{2}$ and \\ Josep M. Llovet ${ }^{1,2,5}$
}

${ }^{1}$ Liver Cancer Translational Research Liver Cancer Translational Research Group, Institut d'Investigacions Biomèdiques August Pi i Sunyer (IDIBAPS)-Hospital Clínic, Liver Unit, Universitat de Barcelona, Barcelona, Catalonia, Spain; ${ }^{2}$ Mount Sinai Liver Cancer Program, Department of Liver Diseases, Icahn School of Medicine at Mount Sinai, New York, USA; ${ }^{3}$ Liver diseases, Vall d'Hebron Institut de Recerca (VHIR), Hospital Universitari Vall d'Hebron, Barcelona, Spain; ${ }^{4}$ Division of Chronic Inflammation and Cancer, German Cancer Research Center Heidelberg (DKFZ), Heidelberg, Germany; and ${ }^{5}$ Institució Catalana de Recerca $i$ Estudis Avançats (ICREA), Barcelona, Catalonia, Spain

\section{See Covering the Cover synopsis on page 1176}

BACKGROUND \& AIMS: Cirrhosis and chronic inflammation precede development of hepatocellular carcinoma (HCC) in approximately $80 \%$ of cases. We investigated immune-related gene expression patterns in liver tissues surrounding earlystage HCCs and chemopreventive agents that might alter these patterns to prevent liver tumorigenesis. METHODS: We analyzed gene expression profiles of nontumor liver tissues from 392 patients with early-stage HCC (training set, $\mathrm{N}=167$ and validation set, $\mathrm{N}=225$ ) and liver tissue from patients with cirrhosis without HCC $(\mathrm{N}=216$, controls $)$ to identify changes in expression of genes that regulate the immune response that could contribute to hepatocarcinogenesis. We defined 172 genes as markers for this deregulated immune response, which we called the immune-mediated cancer field (ICF). We analyzed the expression data of liver tissues from 216 patients with cirrhosis without HCC and investigated the association between this gene expression signature and development of HCC and outcomes of patients (median follow-up, 10 years). Human liver tissues were also analyzed by histology. C57BL/6J mice were given a single injection of diethylnitrosamine (DEN) followed by weekly doses of carbon tetrachloride to induce liver fibrosis and tumorigenesis. Mice were then orally given the multiple tyrosine inhibitor nintedanib or vehicle (controls); liver tissues were collected and histology, transcriptome, and protein analyses were performed. We also analyzed transcriptomes of liver tissues collected from mice on a choline-deficient high-fat diet, which developed chronic liver inflammation and tumors, orally given aspirin and clopidogrel or the anti-inflammatory agent sulindac vs mice on a chow (control) diet. RESULTS: We found the ICF gene expression pattern in $50 \%$ of liver tissues from patients with cirrhosis without HCC and in $60 \%$ of nontumor liver tissues from patients with early-stage HCC. The liver tissues with the ICF gene expression pattern had 3 different features: increased numbers of effector T cells; increased expression of genes that suppress the immune response and activation of transforming growth factor $\beta$ signaling; or expression of genes that promote inflammation and activation of interferon gamma signaling. Patients with cirrhosis and liver tissues with the immunosuppressive profile (10\% of cases) had a higher risk of HCC (hazard ratio, 2.41; 95\% confidence interval, 1.21-4.80). Mice with chemically induced fibrosis or diet-induced steatohepatitis given nintedanib or aspirin and clopidogrel downregulated the ICF gene expression pattern in liver and developed fewer and smaller tumors than mice given vehicle. CONCLUSIONS: We identified an immune-related gene expression pattern in liver tissues of patients with early-stage HCC, called the ICF, that is associated with risk of HCC development in patients with cirrhosis. Administration of nintedanib or aspirin and clopidogrel to mice with chronic liver inflammation caused loss of this gene expression pattern and development of fewer and smaller liver tumors. Agents that alter immune regulatory gene expression patterns associated with carcinogenesis might be tested as chemopreventive agents in patients with cirrhosis.

Keywords: Cancer; Microenvironment; Cytokines; Lymphocytes.

T iver cancer is the fourth leading cause of cancerrelated mortality worldwide. ${ }^{1}$ Hepatocellular carcinoma (HCC) accounts for more than $90 \%$ of liver cancers and is the main cause of death in patients with cirrhosis., ${ }^{2,3}$ HCC arises from chronic liver inflammation, fibrosis, and eventually cirrhosis in $70 \%-80 \%$ of cases. $^{2}$ In developed countries, curative treatments are feasible in $30 \%-40 \%$ of

Abbreviations used in this paper: $\mathrm{Cl}$, confidence interval; $\mathrm{CCl}_{4}$, carbon tetrachloride; CD-HFD, choline-deficient high-fat diet; DEN, diethylnitrosamine; EGFR, epidermal growth factor receptor; ERK, extracellular signal-regulated kinase; GSEA, gene set enrichment analysis; HCC, hepatocellular carcinoma; HCV, hepatitis C virus; HSC, hepatic stellate cell; ICF, immune-mediated cancer field; IFN, interferon; IL, interleukin; NK, natural killer; $p$, phosphorylated; ssGSEA, single sample gene set enrichment analysis; STAT, signal transducer and activator of transcription; TGF, transforming growth factor; VEGFR, vascular endothelial growth factor receptor.

Most current article

(C) 2019 by the AGA Institute 0016-5085/\$36.00

https://doi.org/10.1053/j.gastro.2019.07.028 


\section{WHAT YOU NEED TO KNOW \\ BACKGROUND AND CONTEXT}

We investigated inflammatory and immune-related gene expression patterns in liver tissues surrounding earlystage HCCs and chemopreventive agents that might alter these patterns to prevent liver tumorigenesis.

\section{NEW FINDINGS}

We identified a gene signature that associated with risk of HCC development in patients with cirrhosis. Administration of immunomodulatory and antiangiogenic agents to mice with chronic inflammation and fibrosis reduced this gene expression pattern and reduced development of liver tumors.

\section{LIMITATIONS}

This was a retrospective analysis of human liver tissue samples and the chemopreventive agents were tested in mice with liver tumors due to chronic fibrosis or steatosis. More human studies are needed.

\section{IMPACT}

This gene expression signature might be used to identify patients with cirrhosis who are at risk for HCC. Pharmacologic agents that alter this gene expression pattern might be tested in chemoprevention studies.

cases, but recurrence is high, and no effective adjuvant therapies are available. ${ }^{2,4}$ In addition, in approximately $40 \%-50 \%$ of patients, diagnosis is made at advanced stages, when currently approved molecular therapies yield limited survival benefits (approximately 1 year). ${ }^{3}$ Despite recent advances in the management and clearance of hepatits $\mathrm{C}$ virus (HCV) infection, there is an unmet need for early detection and application of chemopreventive approaches in patients at high risk for HCC development.

To date, there are no established preventive strategies for HCC in patients at risk beyond prevention with antiviral therapies. ${ }^{5}$ Once cirrhosis is established, antiviral therapies reduce but do not eliminate the risk of HCC. ${ }^{4,6,7}$ Individual risk assessment is a key first step in the successful development of any chemopreventive strategy. In this regard, increasing evidence suggests the existence of the so-called cancer field effect or field cancerization, which consists of predisposing oncogenic and inflammatory signals occurring during chronic liver injury and ultimately leading to malignant transformation. ${ }^{8-10}$ Gene signatures derived from the cirrhotic tissue adjacent to HCC tumors have been designed to predict poor outcome, particularly in patients with cirrhosis and HCV infection who are at higher risk of HCC development., ${ }^{91-14}$ Overall, these studies support the feasibility of using molecular scores of the carcinogenic field to identify patients at high risk for HCC development. However, the carcinogenic roles of inflammation and immune response in the context of field cancerization have been poorly explored. Understanding the immune features governing the unresolved cancer field effect is crucial for identifying potential therapeutic targets in patients at high risk for HCC development.

In this study, the analysis of the inflammatory milieu that characterizes the underlying liver disease in which
HCC tumors arise has led to the identification of an immune-mediated cancer field (ICF) in $60 \%$ of patients with early HCC and $50 \%$ of patients with cirrhosis but without HCC. This ICF comprises 3 distinct molecular subtypes: the high-infiltrate ICF subtype, with increased infiltration of effector T cells; the immunosuppressive ICF subtype, with activation of stroma and transforming growth factor (TGF) $\beta$ signaling; and the proinflammatory ICF subtype, with upregulation of interferon (IFN) gamma signaling. These immune profiles, particularly the immunosuppressive cancer field, predict increased risk of HCC development in cirrhotic patients. Inhibition of this carcinogenic field significantly reduced HCC onset in 2 mouse models of chronic liver damage and hepatocarcinogenesis. Overall, our study provides the rationale for exploring chemopreventive strategies in patients with cirrhosis at high risk for HCC development.

\section{Materials and Methods}

\section{Human Cohort}

Gene expression data from a cohort of 167 surgically resected fresh-frozen samples (Heptromic data set, GSE63898) with matched tumor and adjacent nontumor tissue were analyzed. Samples were previously collected (1998-2008) in the setting of the HCC Genomic Consortium after institutional review board approval. Full descriptions of the cohort and RNA profiling data are available in previous publications. ${ }^{15,16}$ Supplementary Table 1 provides a summary of the clinicopathologic variables of the samples used in the current study (training cohort, $\mathrm{N}=167$ ). Validation of the identified molecular profiles was then performed in an independent set of 225 adjacent nontumor liver tissues previously characterized by our group (GSE10143). ${ }^{9}$ Finally, to identify those patients without neoplasm at higher risk of HCC development and most likely to benefit from chemopreventive strategies, our findings were evaluated in a previously characterized cohort of patients with early cirrhosis $(\mathrm{N}=216, \mathrm{GSE} 15654)^{14}$ and a publicly available data set of fibrotic liver tissues $\left(\mathrm{N}=124\right.$, GSE84044). ${ }^{17}$

\section{Modeling the Immune-Mediated Cancer Field}

Enrichment scores of 4872 gene sets that represent cell states and perturbations of the immune system (collection C7 of MSigDB, Broad Institute) ${ }^{18}$ were calculated by single-sample gene set enrichment analysis (ssGSEA) in the nontumor liver tissue of the training cohort. Next, unsupervised clustering analysis by the nonnegative matrix factorization ${ }^{19}$ method was performed to identify the presence of an ICF. To characterize the samples presenting an ICF and to identify different ICF subtypes, a second unsupervised clustering was performed with SSGSEA scores obtained for a curated set of gene signatures representative of individual cell types, ${ }^{20,21}$ cancer immune-related signaling pathways, ${ }^{22}$ and inflammation- or immune-specific biological processes (Hallmark collection of MSigDB, Broad Institute).

\section{Generation of an Immune-Mediated Field Gene Signature}

An ICF field gene signature was generated by using the top differentially expressed genes in each molecular group (false 
discover rate, $<0.05$; fold-change $\geq 2$ ) and was then validated in an independent data set with nearest template prediction analysis $(P<.05)$ (Gene Pattern modules). ${ }^{23}$

\section{Molecular Characterization of the Immune- Mediated Cancer Field Subtypes and Identification of Candidate Therapies}

To characterize the ICF subtypes, gene expression signatures (available in MSigDB [Broad Institute] or previously reported [Supplementary Table 2]) were assessed by GSEA, ssGSEA, nearest template prediction, and ingenuity pathway analyses. CIBERSORT ${ }^{20}$ was used to estimate the relative fraction of 22 immune cell types within the leukocyte compartment of nontumor liver tissues. The immunophenoscore algorithm ${ }^{24}$ was used to analyze the major immunogenic determinants. An in silico analysis based on ssGSEA scores of approximately 1230 gene sets (DSigDB) recapitulating targets of approved therapies was also performed for the screening of candidate targeted therapies.

\section{Histologic Evaluation of Infiltrating Inflammation}

Histopathologic analysis was performed in 98 of 167 cases. Specifically, H\&E staining of formalin-fixed, paraffin-embedded tissue section of HCCs and their matched adjacent nontumor livers were evaluated by 2 expert pathologists (Carla Montironi and Manel Sole). The presence of inflammation (portal/septal, interface, pericentral, and lobular) as well as the lymphoid aggregates were assessed in the nontumor liver tissue sections. More details on the histologic evaluation of the samples have been included in Supplementary Material.

\section{Animal Models}

We generated a chemically induced model of HCC and fibrosis in male C57BL/6J mice (Harlan Laboratories, $\mathrm{N}=55$ ) by a single injection of diethylnitrosamine (DEN) followed by weekly dosing with carbon tetrachloride $\left(\mathrm{CCl}_{4}\right)$, as previously described. ${ }^{25}$ Once fibrosis was established, mice were randomly assigned to receive vehicle or nintedanib $(50 \mathrm{mg} / \mathrm{kg}$; Boehringer Ingelheim, Rhein, Germany). Mice were killed at different time points, and liver and tumor tissue samples were collected and processed for histologic, transcriptomic, and protein expression analyses (see Supplementary Material). All experimental procedures were carried out with the approval of the institutional ethical committee of the University of Barcelona and Hospital Clinic of Barcelona. Additionally, liver samples of a choline-deficient high-fat diet (CD-HFD)-fed mouse model reported in a recent study ${ }^{26}$ were collected. A total of 25 samples were processed for transcriptomic profiling, including mice fed a chow diet $(n=5)$ or CD-HFD for 12 months and given vehicle $(n=4)$, aspirin/clopidogrel $(\mathrm{n}=6)$, or sulindac $(\mathrm{n}=10)$.

\section{Statistical Analysis}

All analyses were performed with SPSS software, version 23 (IBM, Armonk, NY) or GraphPad (San Diego, CA) Prism, version 5.00. Correlations for categorical and continuous variables were analyzed by Fisher exact test and Wilcoxon rank-sum test, respectively. The prognostic value of the signatures was assessed with Kaplan-Meier estimates, log-rank test, and Cox regression models. In in vivo studies, the Mann-Whitney $U$ test was used to compare differences in body weights, liver function, tumor number, tumor size, and CD4/CD8-stained area in human samples. The Fisher exact test was performed for analysis of HCC incidence and phosphorylated extracellular signal-regulated kinase (pERK) staining. The Student $t$ test was used to compare the differences in Sirius Red quantification, CD31 staining, CD4/CD8 staining proportion of immune cell infiltrate in mice, and relative gene expression.

\section{Transcript Profiling}

Gene Expression Omnibus accession numbers are from previously deposited data from our group (GSE63898, GSE10143, GSE15654) and others (GSE84044). Newly profiled mice samples are in the Gene Expression Omnibus under accession number GSE125975 and GSE133969.

\section{Results}

\section{Identification of a Novel Immune-Mediated} Cancer Field Effect in Nontumor Liver Tissue of Patients With Early Hepatocellular Carcinoma

To characterize the immune features governing the unresolved cancer field in which new cancers arise, transcriptome-based analysis of a compendium of approximately 5000 annotated immunology-specific gene sets ${ }^{18}$ was performed in the nontumor liver tissue of patients with early-stage HCC. This analysis showed the presence of an ICF in approximately $60 \%$ (98/167) of samples (Figure $1 A$ and Supplementary Figure 1). Specifically, these samples were characterized by enrichment of several gene sets recapitulating the presence of activated immune cells, as well as up-regulation of core signaling pathways involved in immune response (both innate and adaptive) and those involved in the modulation of inflammatory response (ie, interleukin [IL] 2-signal transducer and activator of transcription [STAT] 5, IL-6/STAT3, IL-17, IFN gamma, colonystimulating factor, tumor necrosis factor [TNF] $\alpha$, and TGF- $\beta$ signaling) (Figure $1 A$ and $B$ and Supplementary Figure 1). Moreover, histologic evaluation confirmed that liver tissues with ICF contained a higher frequency of moderate to marked inflammatory infiltrate $74 \%$ in ICF vs $52 \%$ in non-ICF, $P=.034$ ) and lymphoid aggregates $(80 \%$ in ICF vs $55 \%$ in non-ICF, $P=.009$ ) (Figure $1 C$ and $D$ and Supplementary Table 3). Immunostaining for $\mathrm{CD}^{+}$and $\mathrm{CD}^{+}$further confirmed significantly higher levels of T-cell infiltrates in the adjacent livers of patients with the ICF (Supplementary Figure 2A). In contrast, histologic evaluation of the tumor showed no significant correlation between presence of the ICF and detection of intratumoral or peritumoral infiltration (Supplementary Table 3). This is in accordance with our recent publication, ${ }^{15}$ where the tumor immune-based profile did not correlate with the presence or absence of immune gene signatures in the surrounding nontumor tissue.

While characterizing the ICF we detected that, in addition to immunogenic features, several well-known carcinogenic signals, such as epithelial-to-mesenchymal transition, 
KRAS, epidermal growth factor receptor (EGFR), and vascular endothelial growth factor (VEGF) signaling, were also significantly enriched in liver tissues containing the ICF
(Supplementary Table 4). In line with these oncogenic signals, a significant enrichment of previously reported prognostic signatures derived from the adjacent nontumoral

A
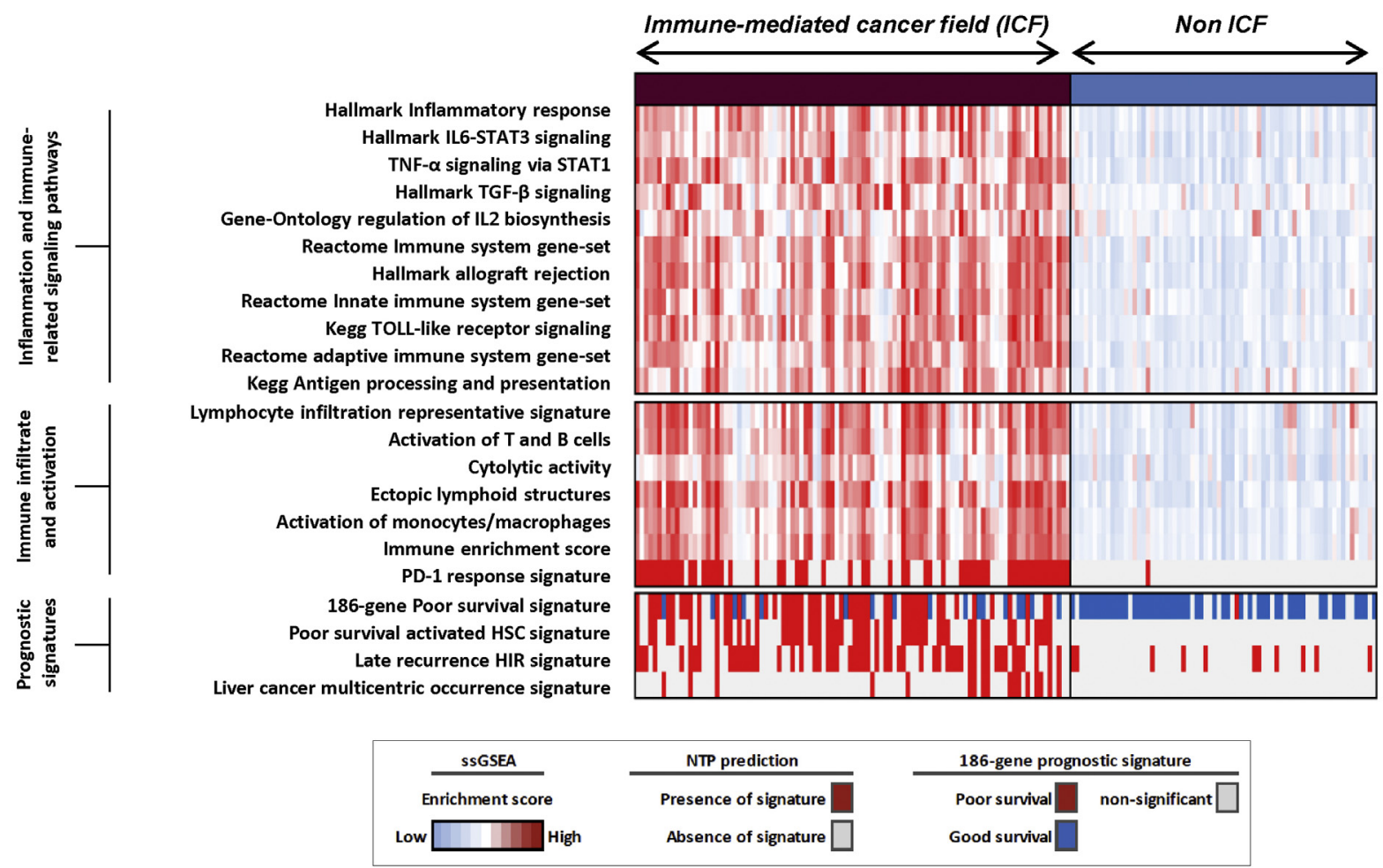

B
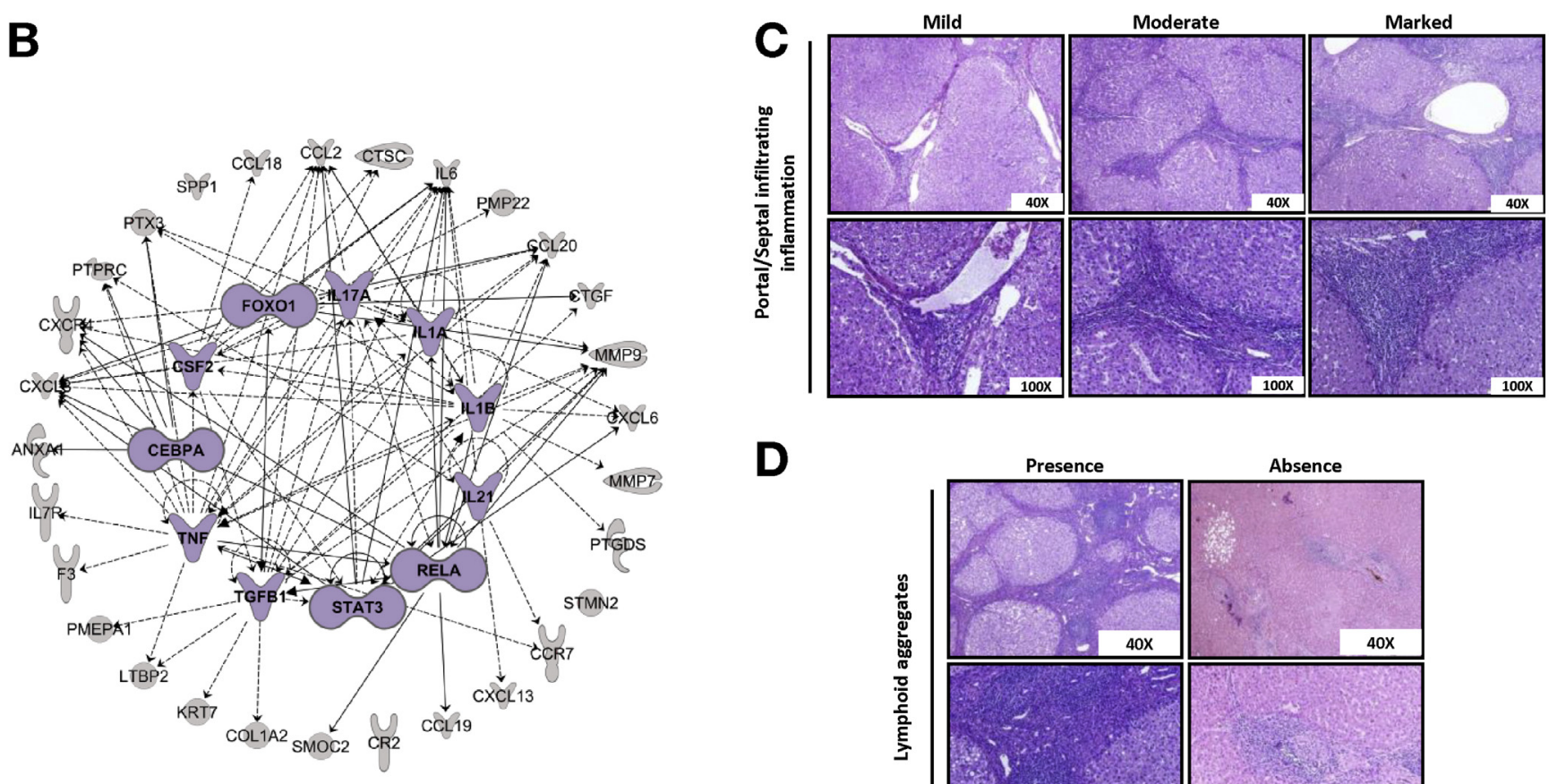

D

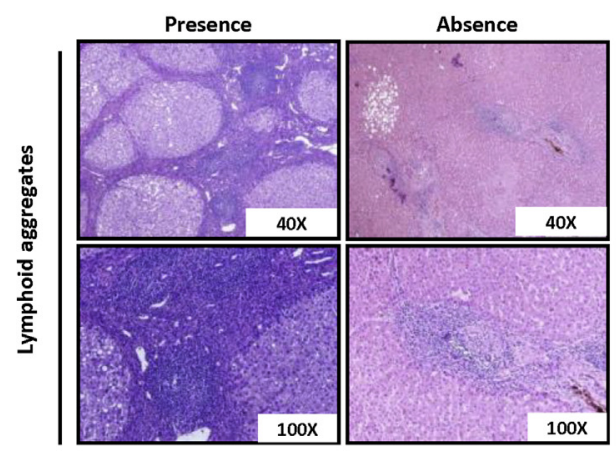

Figure 1. Identification of an ICF effect in nontumoral liver tissue adjacent to early HCCs. $(A)$ Heatmap representation of the ICF present in $60 \%$ of patients with HCC. High and low ssGSEA scores are represented in red and blue, respectively. (B) Top predicted upstream cytokine and transcription factors activated in liver tissues of ICF patients. $(C)$ Representative images of degree of portal/septal infiltrating inflammation. Original magnifications are given. $(D)$ Representative images depicting presence or absence of lymphoid aggregates. Original magnifications are given. HIR, hepatic injury and regeneration; NTP, nearest template prediction; PD-1, programmed cell death protein 1. 
liver were also detected. These signatures included the 186-gene cancer-field signature, ${ }^{9}$ activated hepatic stellate cells (HSCs), ${ }^{11}$ hepatic injury and regeneration, ${ }^{13}$ and multicentric occurrence of $\mathrm{HCCs}^{27}$ (Figure $1 A$ ). The presence of the ICF significantly correlated with HCV infection; features indicative of liver dysfunction such as high bilirubin, low platelet count, and albumin levels (Supplementary Table 5); and poor survival (median overall survival, 43.4 months in the ICF group vs 94.8 months in the non-ICF group; $P=.001$ ], (Supplementary Table 6 and Supplementary Figure $1 B$ ). Altogether, our data highlight the presence of an ICF in $60 \%$ of patients with early HCC. This ICF is characterized by activation of immunomodulatory signaling cascades (ie, IFN- $\gamma$, TNF- $\alpha$, TGF- $\beta$, and IL-6), along with cancer-promoting signaling pathways (ie, epithelialmesenchymal transition, EGFR, and vascular endothelial growth factor receptor [VEGFR]), and is associated with HCV infection and poor prognosis.

\section{The Immune-Mediated Cancer Field Contains 3 Distinct Molecular Subtypes}

Further dissection of the key immune-modulating signaling pathways and immune-cell infiltrates in those samples harboring the ICF showed the existence of 3 distinct molecular subtypes. The first molecular subtype, the high-infiltrate ICF subtype (23\% of the ICF), showed a significant enrichment of several previously established gene signatures mirroring the presence and/or activation of immune cell infiltrates such as lymphocytes ( $\mathrm{T}$ and B cells) ${ }^{22,28}$ or macrophages ${ }^{29}$ (Figure $2 A$ and $B$ ). Consistently, immunogenicity, herein captured either by the cytolytic activity score (Figure $2 A)^{30}$ or by using the immunophenoscore algorithm $^{24}$ (Figure $2 B$ ), was also significantly higher in these samples $(P<.001)$. Specifically, nontumor liver samples belonging to the high-infiltrate ICF subtype showed significant infiltration of effector $\mathrm{T}$ cells (Figure $2 B)(P \leq .001)$, including increased levels of cytotoxic $\mathrm{CD}^{+} \mathrm{T}$ cells assessed both by transcriptomic $(P=.03)$ and immunohistochemistry $(P=.0002)$ (Figure $2 C$ and Supplementary Figure $2 B$ ). This subtype also was characterized by enrichment of the previously reported ectopic lymphoid structures signature $^{31}$ (Figure 2A). In addition, the high-infiltrate ICF was significantly associated with poor survival compared with the rest of the patients (Supplementary Figure 1C), although there were no significant differences among the distinct ICF subtypes (Supplementary Figure 1D). The second subtype, the immunosuppressive ICF (36\% of the ICF), was characterized primarily by activation of stroma and HSCs, increased TGF- $\beta$ signaling, and T-cell exhaustion (Figure 2A). Moreover, several immune checkpoints (ie, CTLA-4, TIGIT, LAG3) were significantly overexpressed (immunophenoscore, $P<.01$ ) in this class, along with higher levels of M2 macrophages $(P=.04)$ and $C D 4^{+}$ memory resting cells $(P=.005)$, which are among the main mediators of immune tolerance and inhibition (Figure $2 B$ and $C$ ). The third subtype ( $41 \%$ of the ICF) showed a clear predominance of IFN gamma signaling $(P<.001)$ and enrichment of the inflammatory M1 macrophages
$(P<.0001)$; it is called the proinflammatory ICF subtype (Figure $2 A-C$ ). The high-infiltrate and immunosuppressive subtypes shared several molecular features, including the enrichment of key signaling pathways involved in modulating the immune response (ie, IL-2 and TNF signaling), proliferation (ie, KRAS signaling), and angiogenesis $(P<.001)$ (Figure $2 A)$.

To further confirm the presence and molecular traits of the identified ICF, we generated a transcriptome-based gene signature able to capture the 3 ICF subtypes. This signature showed only minimal overlap $(0 \%-5 \%)$ with previously reported gene signatures of field cancerization in HCC (Supplementary Figure 3). 9,12,14,32 The resulting 172-gene signature (Supplementary Table 7) was then validated in the adjacent nontumor tissue of 225 patients with early HCC, previously characterized by our group ${ }^{9,33}$ (Supplementary Figure $4 A$ ). Similar to what was previously observed in the training cohort, 58\% (130/225) of patients belonged to the ICF. Moreover, in this cohort, the presence of the ICF was an independent predictor of poor survival (hazard ratio, 2.73; 95\% confidence interval [CI], 1.1-6.8; $P=.03$ ) (Supplementary Figure $4 B$ and Supplementary Table 8). Within the ICF group, approximately $31 \%(40 / 130)$ presented the high-infiltrate ICF, approximately $27 \%(35 / 130)$ the immunosuppressive ICF, and approximately $42 \%$ (55/130) the proinflammatory ICF subtype (Supplementary Figure $4 A$ ). Subsequent molecular characterization further confirmed the ability of the signature to capture the main molecular traits defining each subtype, such as increased infiltration of effector $\mathrm{T}$ cells in the high-infiltrate subtype, activation of stroma and TGF- $\beta$ signaling in the immunosuppressive subtype, and up-regulation of IFN gamma signaling in the proinflammatory subtype (Supplementary Figure 4A). Overall, our results highlight the presence of a poor prognosisrelated ICF comprising 3 molecular subtypes with a high degree of lymphocyte infiltration (overall, 16\% of patients with HCC) or predominance of either immunosuppressive (overall, $20 \%$ of patients with HCC) or proinflammatory (overall, $24 \%$ of patients with HCC) signaling cascades.

\section{The Immune-Mediated Cancer Field, Particularly the Immunosuppressive Subtype, Predicts a High Risk of Hepatocellular Carcinoma Development in Patients With Cirrhosis}

After the identification of an ICF in the livers of $60 \%$ of patients with early HCC, we next sought to assess its role in liver disease progression and HCC primary occurrence. To this end, the 172-gene signature was analyzed in a cohort of 216 patients with nonmalignant cirrhosis with a median follow-up of 10 years in the context of an HCC surveillance program. $^{14}$ Overall, $51 \%(110 / 216)$ of patients with cirrhosis harbored the ICF, including the high-infiltrate ICF subtype in $28 \%(31 / 110)$, the immunosuppressive ICF subtype in $19 \%(21 / 110)$, and the proinflammatory ICF subtype in 53\% (58/110) of patients with cirrhosis harboring the ICF. 
A

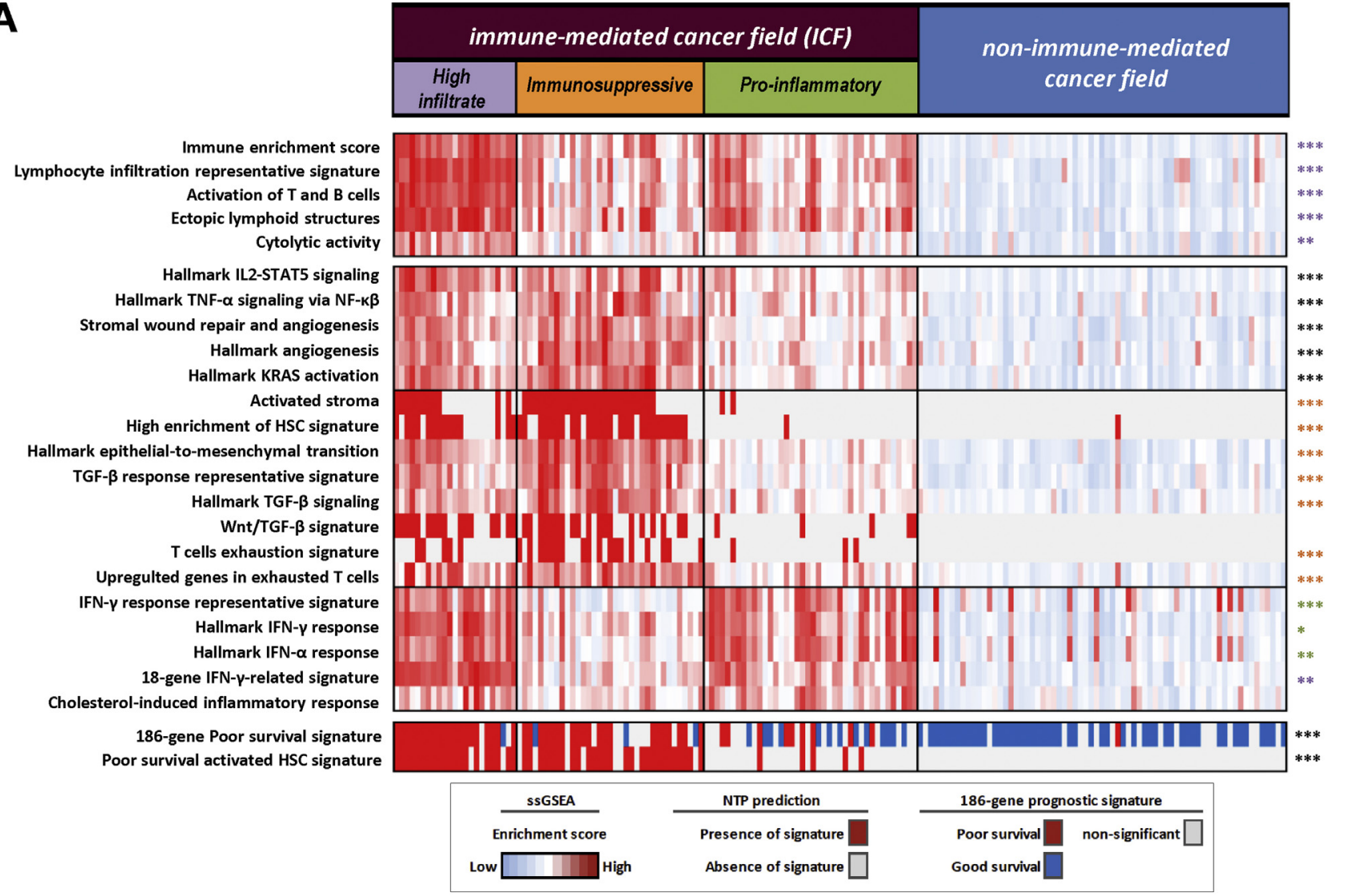

B
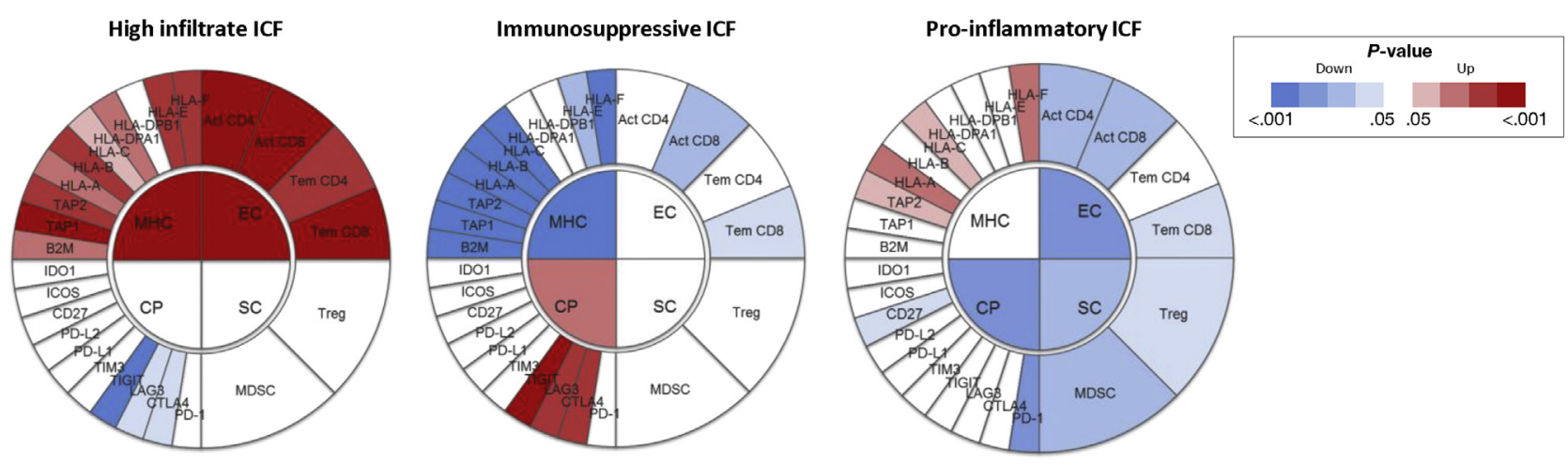

C
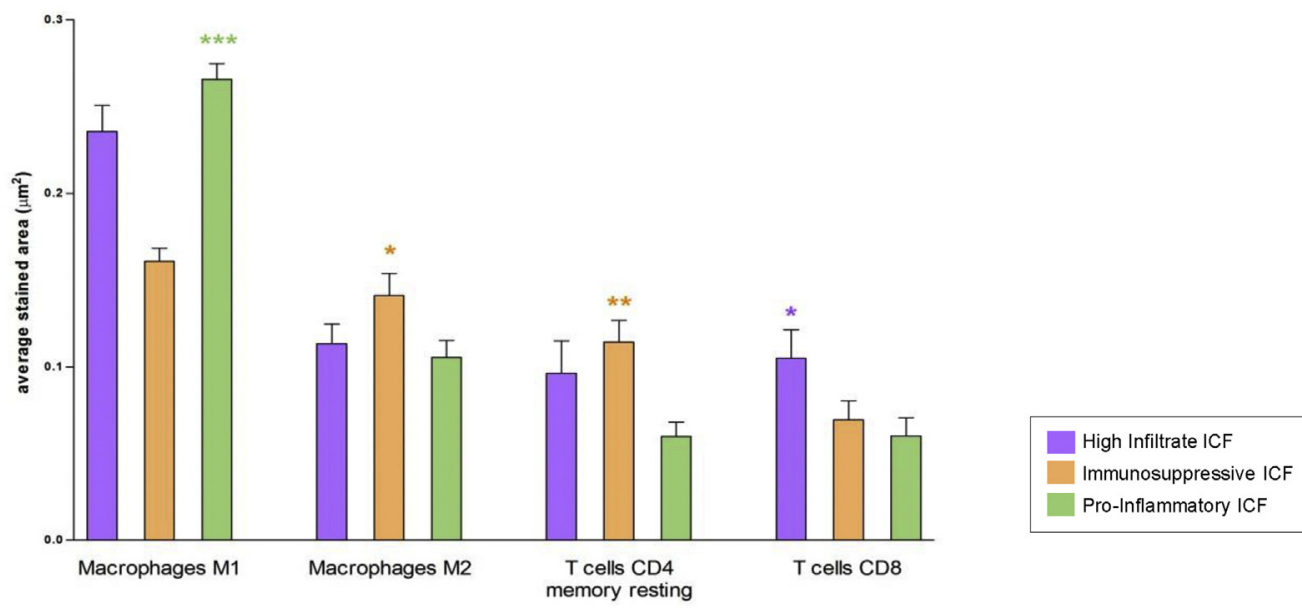
Next, we tested the capacity of the ICF subtypes to predict the risk of HCC development in patients with cirrhosis. The presence of the immunosuppressive ICF subtype $(10 \%$ of all patients with cirrhosis) was significantly associated with a higher risk of HCC development (median time to HCC development of 7.4 years [95\% CI, 3.211.7] vs 17.1 years [95\% CI, 10.6-23.7] in Rest, $P<.0001$ ] and was found to be an independent predictor of HCC occurrence in patients with cirrhosis in a multivariate analysis (hazard ratio, 2.41; 95\% CI, 1.2-4.8; $P=.012$ ) (Figure $3 A$ and Table 1 ). In addition, the immunosuppressive ICF was also significantly associated with poor survival (median overall survival, 7.1 years [95\% CI, 4.5-9.6] vs 16.3 years [95\% CI, 9.1-23.5] in Rest; $P<.0001$ ) and higher risk of hepatic decompensation (median time to hepatic decompensation, 6.5 years [95\% CI, 4.3-8.6] vs $>15$ years in Rest; $P<.0001$ ) (Figure $3 B$ and $C$ ). Patients with cirrhosis harboring the high-infiltrate and proinflammatory ICF subtypes also showed a nonsignificant trend toward a higher risk of HCC development compared with those patients lacking the ICF (mean time to HCC development, 12.8 years [95\% CI, 11.5-14.2] in other ICF subtypes vs 16.3 years [95\% CI, 14.2-18.5] in non ICF, $P=.06$ ) (Supplementary Figure $5 A$ ).

Moreover, the analysis of an additional cohort of 124 patients without neoplasia with liver fibrosis ${ }^{17}$ showed that the ICF may occur as a progressive event because it was significantly correlated with increasing levels of fibrosis stage and degree of inflammation (Supplementary Figure 5B). Particularly, the presence of the immunosuppressive ICF was significantly correlated with the presence of advanced liver fibrosis (Scheuer fibrosis S3-4 score, ${ }^{17} P=$ .034) (Supplementary Figure 5B).

In conclusion, the ICF detected in patients with early HCC is also present in the livers of approximately $50 \%$ of patients with cirrhosis and captures the presence of a damaging and continuous inflammatory response in the underlying liver disease. Furthermore, our results underscore the critical role of an immunosuppressive ICF (overall, $10 \%$ of patients with cirrhosis) in defining a 2.4-times risk of HCC development and, to a smaller extent, of the highinfiltrate and proinflammatory subtypes.

\section{The Immune-Mediated Field as a Target for Chemoprevention in a Mouse Model Recapitulating Chronic Liver Inflammation and Hepatocellular Carcinoma Development}

Based on the compelling results described, we hypothesized that the ICR, particularly the
A

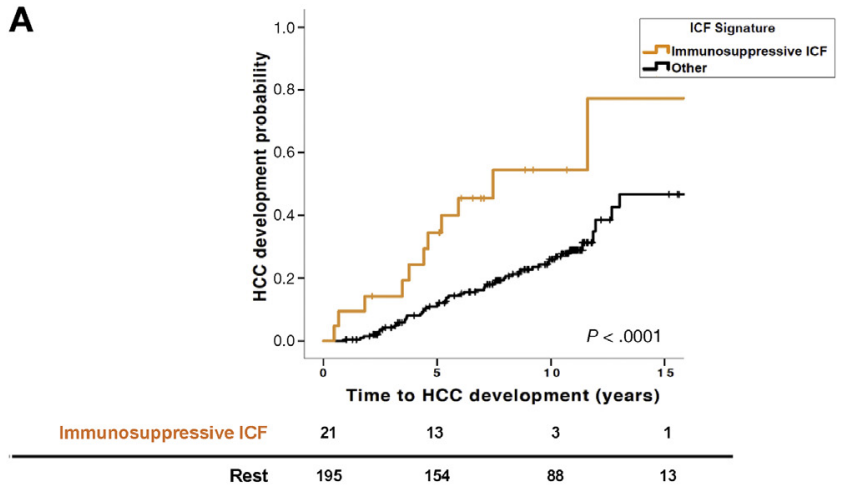

B
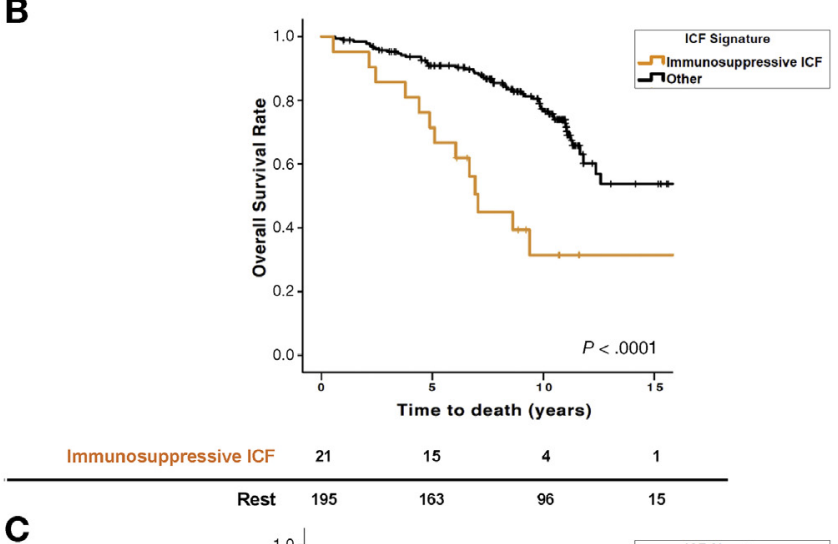

C

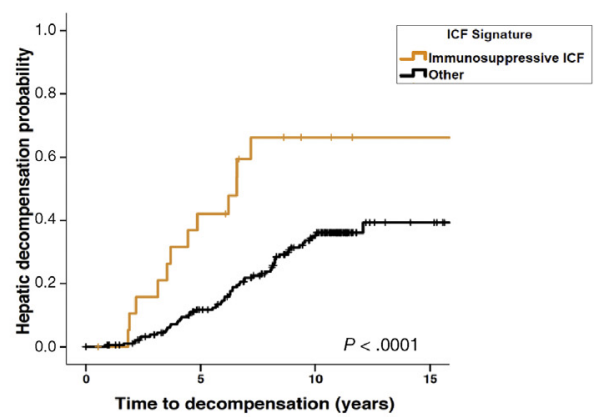

Immunosuppressive ICF $\quad 21$

$\begin{array}{lllll}\text { Rest } & 195 & 152 & 78 & 14\end{array}$

Figure 3. Association of the presence of the immunnosuppressive ICF with HCC occurrence and prognostic variables in patients with cirrhosis. (A) Kaplan-Meier estimates of HCC development, $(B)$ overall survival, and $(C)$ hepatic decompensation, according to the presence of the immunosuppressive ICF subtype (orange).

immunosuppressive ICF subtype, may represent an ideal target for chemopreventive strategies in patients with cirrhosis at high risk for HCC development. To this purpose, an in silico-based analysis was performed with our training cohort to identify those candidate therapies most

Figure 2. The ICF contains 3 distinct molecular subtypes. $(A)$ Heatmap representation of the 3 ICF subtypes. Statistical significance is highlighted. $(B)$ Immunophenogram representing the enrichment of immunogenic determinants in the distinct ICF subtypes $(C)$ Comparison of estimated proportion of immune cells (CIBERSORT method) between the ICF subtypes, representing those immune populations with estimated average fraction $>5 \%$ and significant differences between the ICF subtypes. Significant statistical differences observed among the different ICF subtypes are highlighted (high infiltrate, purple; immunosuppressive, orange; proinflammatory, green; and both high infiltrate and immunosuppressive, black). ${ }^{\star} P<.05$, ${ }^{\star \star} P<$ .01 , and ${ }^{\star \star \star} P<.001$. CP, checkpoint, EC, effector cell; MDSC, myeloid-derived suppressor cell; MHC, major histocompatibility complex in antigen presenting cells; NTP, nearest template prediction; SC, suppressor cells; Treg, regulatory T cell. 
Table 1.Univariate and Multivariate Analysis of Risk of Hepatocellular Carcinoma Development in Patients With Cirrhosis Including Gene Signatures and Clinicopathologic Variables $(\mathrm{N}=216)$

\begin{tabular}{|c|c|c|c|c|}
\hline \multirow[b]{2}{*}{ Variable } & \multirow{2}{*}{$\frac{\text { Univariate analysis }}{P \text { value }}$} & \multirow[b]{2}{*}{ Hazard ratio } & \multicolumn{2}{|c|}{ Multivariate analysis (Cox regression) } \\
\hline & & & $95 \% \mathrm{Cl}$ & $P$ value \\
\hline \multicolumn{5}{|l|}{ Nontumoral liver tissue-based transcriptomic profiles } \\
\hline Immunosuppressive-ICF & .03 & 2.41 & $1.21-4.80$ & .01 \\
\hline 186-gene poor prognosis signature & $<.0001$ & 1.56 & $0.89-2.7$ & .12 \\
\hline \multicolumn{5}{|l|}{ Clinicopathologic variables } \\
\hline Age ( $>$ median) & .87 & & & \\
\hline Sex & .22 & & & \\
\hline Diabetes & .48 & & & \\
\hline HCV genotype $1 b$ & .18 & & & \\
\hline Alcohol consumption (>80 g/day) & .68 & & & \\
\hline HCV etiology plus alcohol consumption & .68 & & & \\
\hline History of antiviral treatment (IFN based) & .65 & & & \\
\hline Varices & .02 & 1.49 & $0.85-2.6$ & .17 \\
\hline Spleen & .13 & & & \\
\hline Ishak score of 6 vs 5 & .24 & & & \\
\hline Platelet count $\left(<100,000 / \mathrm{mm}^{3}\right)$ & .02 & 1.51 & $0.91-2.64$ & .15 \\
\hline Bilirubin $(>1 \mathrm{mg} / \mathrm{dL})$ & $<.01$ & 1.85 & $1.07-3.2$ & .03 \\
\hline Alpha-fetoprotein $(>10 \mathrm{ng} / \mathrm{mL})$ & .87 & & & \\
\hline Prothrombin time (international normalized ratio $>1.2$ ) & .38 & & & \\
\hline
\end{tabular}

NOTE. Text in bold font indicates statistical significance.

likely to modulate the identified ICF. This analysis was based on the enrichment of a compendium of approximately 1230 gene sets (DSigDB collections D1 and D2) ${ }^{34}$ recapitulating the main targets of 1202 approved drugs. Among the top 10 most significantly enriched drugs (Supplementary Figure 6), nintedanib was the only therapy approved by the US Food and Drug Administration indicated for a noncancer condition. Specifically, nintedanib is the first molecular targeted therapy with clinical efficacy in patients with idiopathic pulmonary fibrosis as both an anti-fibrogenic and anti-inflammatory agent. ${ }^{35}$ Given these considerations, the efficacy of nintedanib in reverting the pro-tumorigenic ICF was tested in a mouse model of HCC development in the setting of chronic inflammation and liver fibrosis (Supplementary Figure $7 A$ ). In this model, the macroscopic evaluation of explanted livers in $\mathrm{DEN} / \mathrm{CCl}_{4}$ mice killed at the ages of 15 , 17 , and 18 weeks confirmed the development of numerous hepatic tumors (Figure $4 A$ ). Tumor penetrance and number of tumors progressively increased, ultimately reaching a $100 \%$ incidence at 18 weeks of age (Figure $4 A$ and $B$ ). At all time points, histologic evaluation of the liver sections showed that a portion of the tumors were preneoplastic (dysplastic) nodules (Figure $4 C$ ). In mice killed at 15 weeks of age (Supplementary Figure $7 A$ ), nintedanib showed a clear trend toward reducing HCC incidence, number, and size of tumors (Figure $4 B, D$, and $E$ ). These differences reached significance at 17 weeks of age (Figure 4B), having a marked decrease in both overall tumor burden $30 \%$ in nintedanib vs $89 \%$ in vehicle group, $P=.019)$ and, specifically, in HCC incidence (7\% vs $33 \%, P=.04)$. Similarly, at 18 weeks of age, HCC incidence was significantly reduced in treated mice $(22 \%$ vs $77 \%, P$ $<$.001) (Figure 4B). In addition, nintedanib significantly reduced the overall tumor number and size both at 17 and 18 weeks of age (Figure $4 D$ and $E$ ). Overall, nintedanib was well tolerated with no significant induction of body weight loss or hepatotoxicity measured by serum alanine aminotransferase and aspartate aminotransferase levels (Supplementary Figure $7 B$ and $C$ ). Taken together, our data suggest that nintedanib is safe and efficacious in preventing HCC development in our experimental model.

\section{Nintedanib Treatment Reverts the Immune- Mediated Cancer Field Effect}

Next, we sought to assess the impact of nintedanib treatment on the ICF. For this purpose, we analyzed gene expression profiling of nontumor liver samples from 17-week-old $\mathrm{DEN} / \mathrm{CCl}_{4}$ mice given nintedanib $(\mathrm{n}=10)$ or vehicle $(\mathrm{n}=9)$ and 3 healthy control mice. First, the comparison between the healthy control and vehicle groups showed a profile of activated pathways compatible with HCC development within a fibrotic and inflammatory background. In this regard, functional analysis of differentially expressed genes (Supplementary Table 9) highlighted the activation of hepatic stellate cells and fibrogenesis, as well as immune system activation (inflammatory response, chemotaxis, binding of myeloid and leukocytes) in vehicletreated $\mathrm{DEN} / \mathrm{CCl}_{4}$ livers (Supplementary Table 10). Notably, our model presented a significant enrichment of the gene set representing the ICF identified in humans $(P=.001)$ and faithfully recapitulated the human ICF 
A

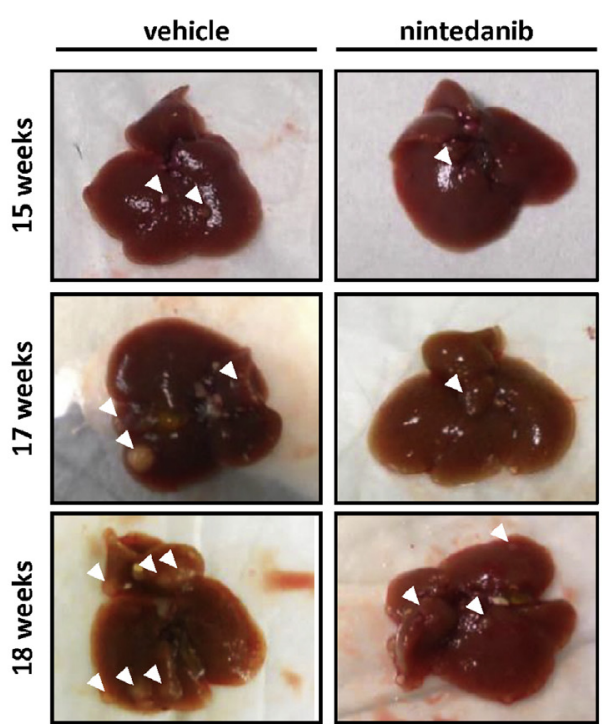

C

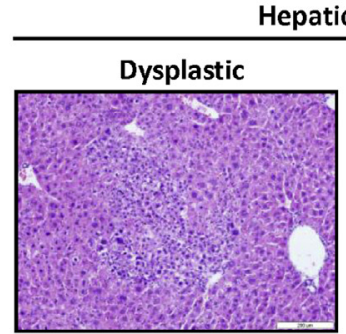

Hepatic tumors

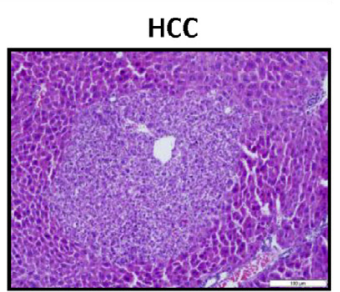

\section{D}
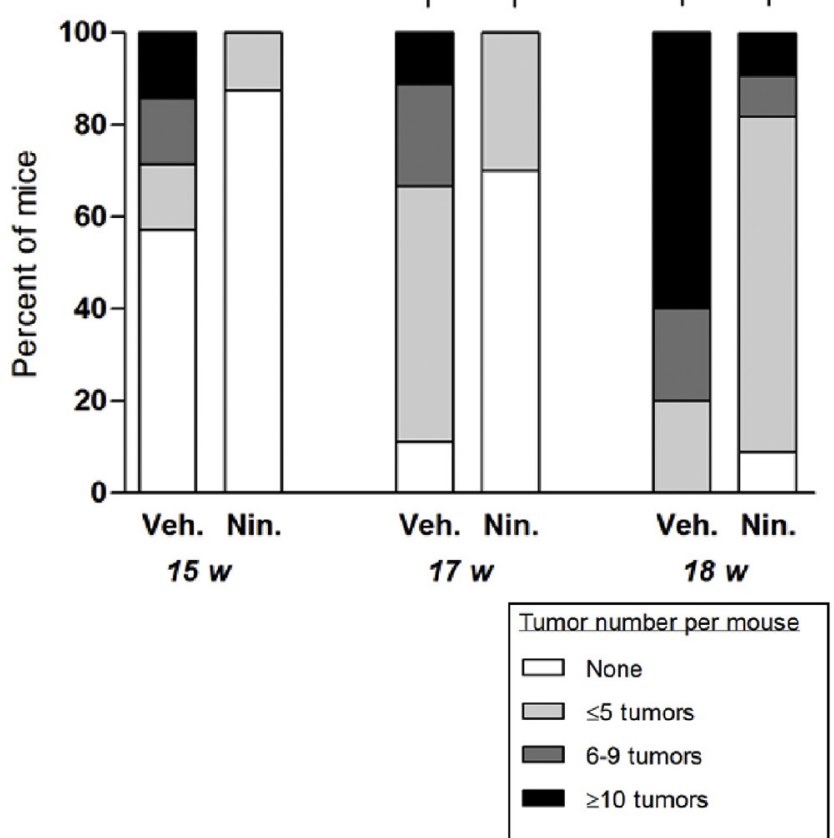
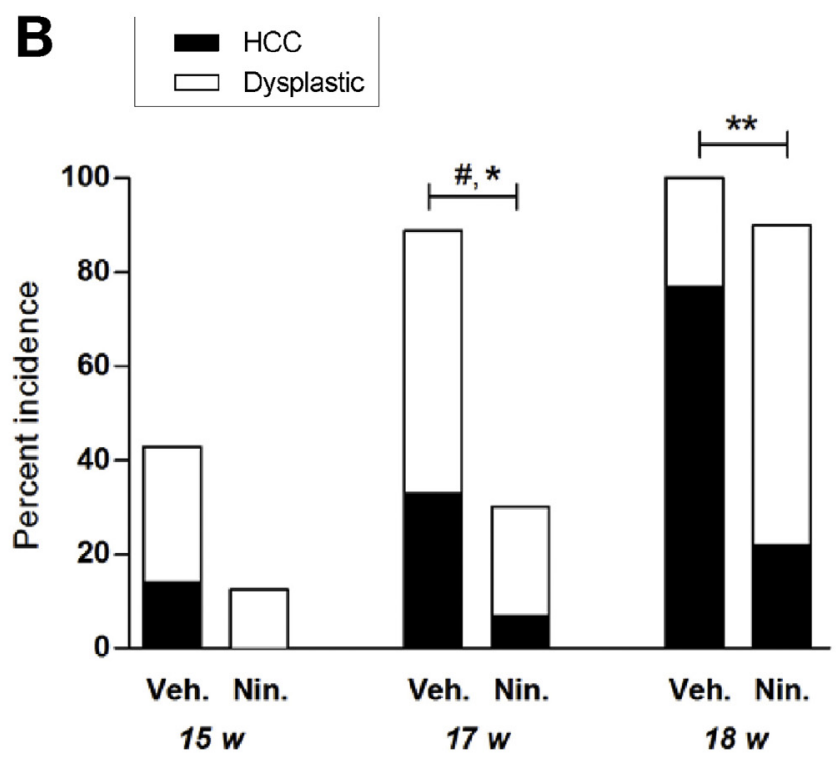

Histological characteristic of HCC

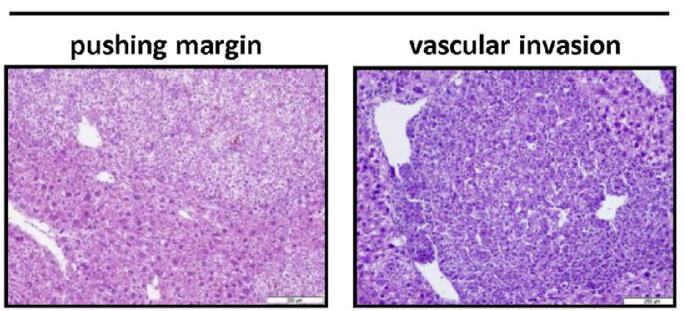

E

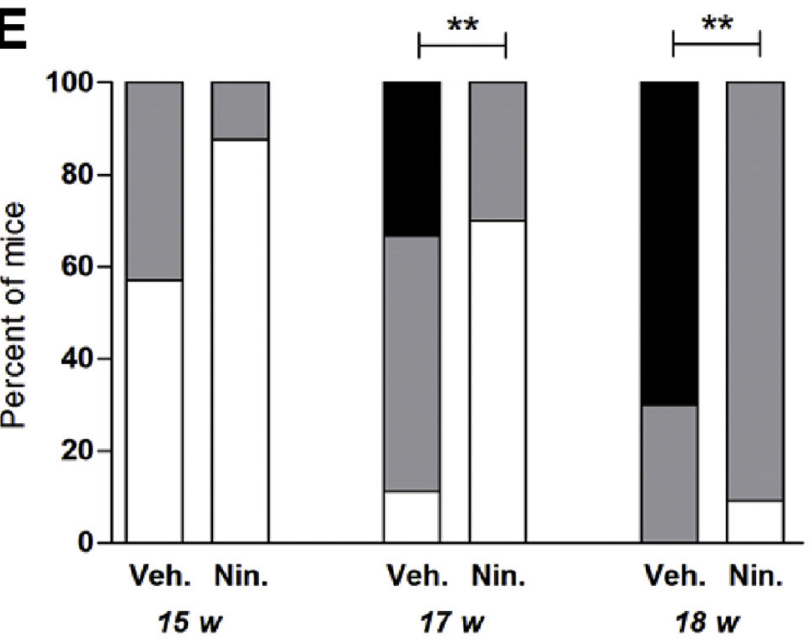

Figure 4. Nintedanib reduces HCC onset in mice. $(A)$ Representative pictures of macroscopic evaluation of hepatic tumors in mice given vehicle or nintedanib and killed at 15, 17, and 18 weeks of age. Arrows indicate macroscopically visible tumors. $(B)$ Evaluation of overall tumor burden and HCC incidence. "Statistical significance for overall tumor burden. ${ }^{*}$ Statistical significance for HCC incidence. (C) Microscopic evaluation of the number of tumors per mouse in each group. (D) Number of macroscopic tumors per mouse given vehicle or nintedanib at the different time points. (E) Diameter size of the largest tumor per mouse given vehicle or nintedanib at the 3 different time points. ${ }^{\#}$ or ${ }^{\star} P<.05$, ${ }^{\# \#}$ or ${ }^{\star \star} P<.01$, and ${ }^{\# \# \#}$ or ${ }^{\star \star \star} P<.001$. Nin, nintedanib; Veh, vehicle; w, weeks. 


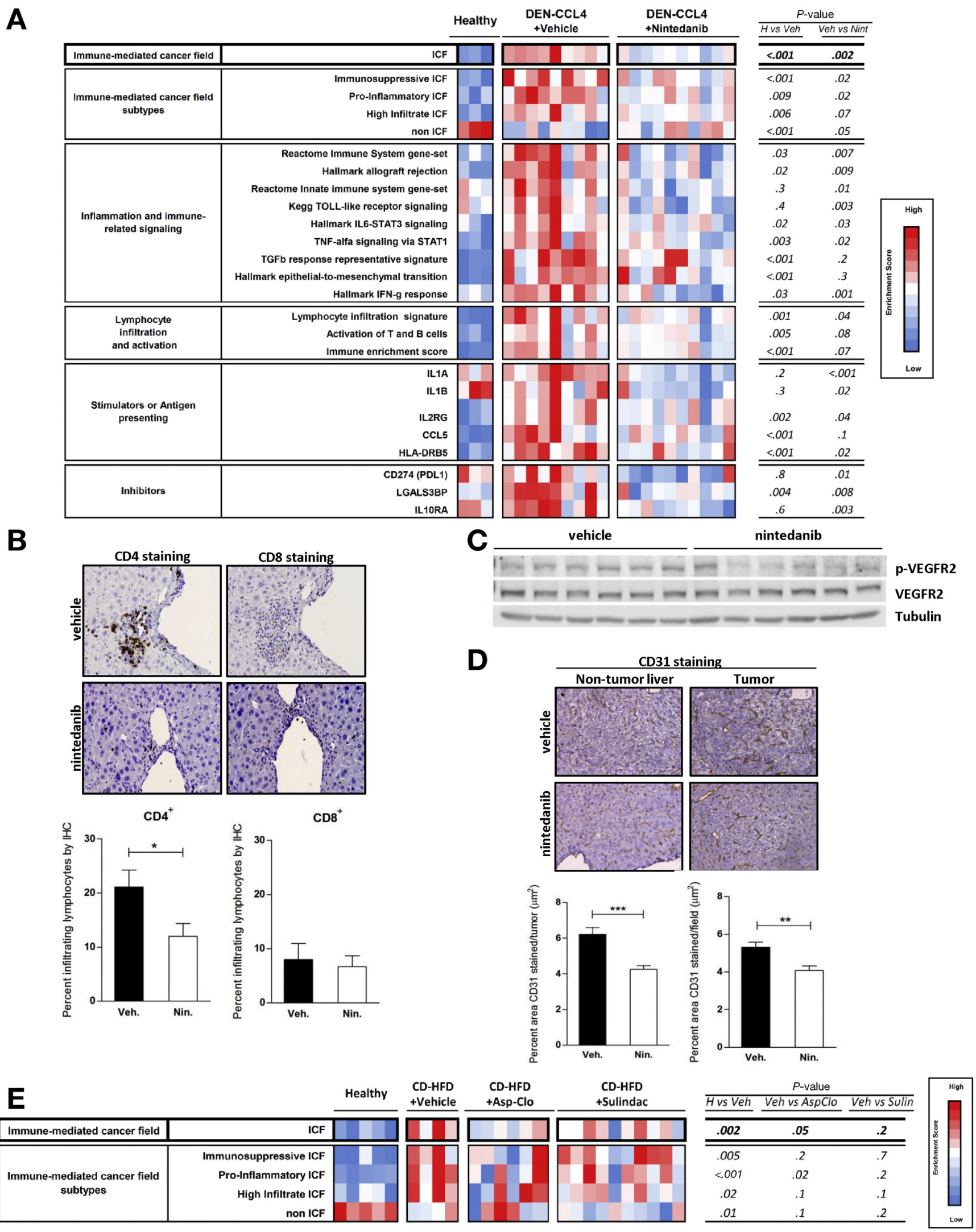


subtypes described (Figure $5 A$ ). The comparison of the gene expression profiles of adjacent nontumor liver from mice treated with vehicle or nintedanib showed that nintedanib significantly down-regulated the ICF subtypes and, more specifically, the proinflammatory and immunosuppressive ICF phenotypes, which predict risk of HCC development in patients with cirrhosis $(P=.02)$ (Figure 5A). A nonsignificant trend was also observed for the high-infiltration subtype (Figure $5 A$ ). Treatment with nintedanib led to a significant down-regulation of inflammatory cues (IL-6/STAT3, IFN alfa, and IFN gamma) and immune-related signaling (IL-2/STAT5 activation, allograft rejection) (Figure $5 \mathrm{~A}$ ). Among the infiltrating immune cells, nintedanib significantly reduced the presence of $B$ and $\mathrm{T}$ cells, activated macrophages, helper $\mathrm{T}$ cells, and regulatory $\mathrm{T}$ cells along with associated immune modulators (ie, IL1, CCL5, and PDL1) (Figure 5A). Despite exhibiting similar global levels of inflammatory infiltrates, quantification of CD4- and CD8-positive infiltrating lymphocytes by immunohistochemistry showed a significant decrease of $\mathrm{CD}^{+} \mathrm{T}$ cells in nintedanib-treated mice compared with controls $(P<.05)$ (Figure $5 B)$.

Next, to further characterize the chemopreventive effects of nintedanib, we assessed the activation status of the main nintedanib targets (ie, VEGFR2 and plateletderived growth factor receptor $\beta$ ). Western blot of nontumor liver tissue confirmed that nintedanib blocked the activation of VEGFR2 (Figure $5 C$ ) and its downstream effectors protein kinase $B$ and ERK (Supplementary Figure $8 A$ ). Consistently, both liver parenchyma and liver tumors were pERK positive in vehicle-treated mice and pERK negative in nintedanib-treated mice $(P<.05)$ (Supplementary Figure $8 B$ ), indicating an antiproliferative effect of nintedanib as well. Given the strong inhibition of VEGFR signaling observed, we next assessed the antiangiogenic effect of nintedanib in $\mathrm{DEN} / \mathrm{CCl}_{4}$ mice. In this model, reduced $\mathrm{CD} 31$ staining was associated with diminished blood vessel area in both liver parenchyma and liver tumors of nintedanib-treated mice (Figure $5 D$ ). Together, these data suggest that nintedanib exhibits its chemopreventive effects in part by inducing vascular normalization and inhibiting hepatic proliferation. In contrast, no reduction of fibrosis degree, the profibrogenic signaling pathway platelet-derived growth factor receptor $\beta$, or collagen markers were detected in the livers of nintedanib-treated mice (Supplementary Figure $8 C-E$ ).

Overall, our data confirm that therapeutic targeting of the ICF, accompanied by liver vascular normalization and suppression of hepatic proliferation, can prevent the development of HCC associated with advanced chronic liver disease.

\section{Immunomodulatory Effects of Aspirin/ Clopidogrel Treatment Revert the Immune- Mediated Cancer Field Effect and Prevent Hepatocarcinogenesis In Vivo}

To further support the concept of an ICF in promoting HCC development and its therapeutic immunomodulation as a candidate strategy for chemoprevention, we performed gene expression profiling in nontumor liver derived from the recently described mouse model of CDHFD treated either with the immunomodulatory combination aspirin/clopidogrel or the anti-inflammatory sulindac. ${ }^{26}$ Of particular interest, in this model, which presents nonalcoholic fatty liver disease-related liver inflammation with various degrees of fibrosis and HCC development after 12 months of diet regimen, ${ }^{26,36,37}$ HCC prevention was achieved only through the combination of the anti-inflammatory drug, aspirin, and clopidogrel (an P2Y12 inhibitor) (25\% vs $0 \% 12$-month HCC incidence, control vs combination, respectively; $P=.01)^{26}$ and not sulindac alone $(25 \%$ vs $20 \% 12$-month HCC incidence, control vs sulindac, respectively; $P=$ not significant; data not shown).

In the context of our study, comparative analysis between the nontumor liver of healthy control and CD-HFD mice showed a significant enrichment of the ICF signature in CD-HFD mice $(P=.002)$ (Figure $5 E$ ). All 3 ICF subtypes were significantly up-regulated in CD-HFD mice compared with healthy controls (Figure $5 E$ ), along with the enrichment of signaling pathways regulating inflammation (ie, IL6/STAT3, TNF- $\alpha$ ), immune infiltration and activation, ${ }^{22,28}$ and epithelial-to-mesenchymal transition (ie, TGF- $\beta)(P<.05)$. These data were consistent with the high intrahepatic influx of metabolically activated $\mathrm{CD}^{+} \mathrm{T}$ cells and natural killer $(\mathrm{NK})$ cells $\left(\mathrm{CD}^{+}{ }^{+} \mathrm{NK} 1.1^{+}\right)$measured in CD-HFD-fed mice by flow cytometry. ${ }^{26}$ Overall, these data confirm the existence of an ICF in an independent model of chronic liver disease, further suggesting a role in hepatocarcinogenesis.

Next, we compared the expression profiles of liver samples from CD-HFD vehicle-treated mice with CD-HFD mice treated with the combination aspirin/clopidogrel $(\mathrm{n}=6)$ or sulindac alone $(\mathrm{n}=10)$. Only aspirin/clopidogrel, but not sulindac alone, was able to prevent HCC and revert the ICF within the liver microenvironment $(P=.05)$, with the proinflammatory ICF subtype being the most significantly down-regulated upon treatment (Figure $5 B$ ).

Figure 5. Nintedanib and aspirin/clopidogrel reduce the ICF in animal models of chronic inflammation and HCC development. (A) Heatmap representation of high and low ssGSEA scores for the 172-gene signature and gene sets recapitulating the ICF subtypes. $(B)$ Representative images and quantification of $\mathrm{CD}^{+}$and $\mathrm{CD}^{+}$infiltrating lymphocytes in the livers of 17 -week-old mice given vehicle $(n=5)$ or nintedanib $(n=5)$. $(C)$ Western blot analysis of VEGFR2 activation in the nontumor liver parenchyma of 17 -week-old mice given vehicle $(n=6)$ or nintedanib $(n=6)$. (D) Morphometric quantification of blood vessel area by CD31 immunostaining in 5 randomly selected, low-magnification fields in mice given vehicle $(n=5)$ or nintedanib $(n=5)$. $(E)$ Single sample GSEA analysis of the ICF signature in the different treatment arms of the CD-HFD model. ${ }^{\star \star} P<.01$ and ${ }^{\star \star \star} P<$ .001. AspClo, aspirin/clopidogrel; Nin., nintedanib; Sulin, sulindac; Veh., vehicle. 
Particularly, based on previous molecular characterization, $^{26}$ the inhibition of the ICF seemed to be accompanied by a significant reduction in the degree of liver damage and a significantly reduced number of $\mathrm{CD}^{+}$and $\mathrm{NK} \mathrm{T}$ cells in the liver.

Overall, these data support the role of the ICF in promoting carcinogenesis and suggest that only those drugs able to simultaneously inhibit several components of the ICF by targeting mitogenic, angiogenic, and immunomodulatory kinases (ie, nintedanib and aspirin/clopidogrel) present a more efficacious therapeutic index for HCC prevention.

\section{Discussion}

This study represents an in-depth analysis of the inflammatory milieu associated with the field cancerization in the chronically injured liver and investigates its clinical implications for the prediction and prevention of HCC occurrence in patients with cirrhosis.

The role of the cancer field effect in promoting neoplastic transformation has gained much interest in recent years, and currently an altered microenvironment is considered a promoter of cancer. ${ }^{8,10}$ Although under physiologic conditions inflammation is an adaptive response to tissue injury, when the inflammatory stimuli persist, the nonresolved inflammation contributes to carcinogenesis. $^{38,39}$ Along these lines, activation of HSC and certain pathways, such as nuclear factor $\kappa \mathrm{B}$ and TGF- $\beta$ signaling, have been previously associated with liver fibrogenesis and, eventually, neoplastic transformation., ${ }^{9,12}$ With this study, we move beyond the limits of current knowledge and provide a detailed description of the immune microenvironment underlying the field cancerization in the liver. To this end, we first characterized the immune profile of the nontumor liver parenchyma of 392 early HCCs and then investigated its role in predicting HCC development in 216 patients with cirrhosis having long-term surveillance for HCC (median, 10 years). ${ }^{14}$ The analysis showed that up to $60 \%$ of HCCs and $50 \%$ of cirrhotic patients showed a deleterious immune-mediated response in the surrounding tissue, which was associated with impaired liver function, activation of specific oncogenic loops, angiogenesis, and poor survival. Further characterization identified 3 distinct subtypes with different levels of lymphocyte infiltration and activation of either immunosuppressive or proinflammatory traits. In particular, the immunosuppressive ICF subtype (approximately 10\% of patients with cirrhosis) was an independent predictor of HCC development, increasing by 2.4 times the risk of cancer development, whereas both the high-infiltrate and the proinflammatory subtypes showed a trend toward higher risk of HCC occurrence in cirrhosis. The identification of distinct immune subtypes reflects the complex role of the immune system in hepatocarcinogenesis, with both an activated immune response and an exhausted immune microenvironment contributing to create a pro-tumorigenic environment and increase the risk of HCC development. ${ }^{40}$

Reducing the incidence and mortality of HCC requires advances in chemopreventive approaches at preneoplastic stages, in addition to curative treatment options for early lesions. Universal immunization against hepatitis B virus and antiviral therapies against hepatitis B virus and HCV have been associated with greatly reduced HCC risk. ${ }^{2,41,42}$ Once cirrhosis is established, the risk of HCC development remains despite achievement of a sustained virologic response in patients with $\mathrm{HCV}^{6,7}$ In addition, the incidence of other risk factors, such as nonalcoholic steatohepatitis, is dramatically increasing. ${ }^{2}$ Thus, alternative HCC preventive strategies capable of interfering with molecular hepatocarcinogenesis are an unmet need. Furthermore, identifying those patients at high risk of HCC development should enable a cost-effective selection of patients most likely to benefit from chemopreventive approaches. In this scenario, our results are of clinical relevance because the ICF, and specifically the immunosuppressive subtype, may provide a novel companion biomarker to enrich at-risk patients in chemoprevention clinical trials. Given these observations, we then sought to investigate whether the molecular forces driving such cancer fields could serve as targets for chemopreventive strategies. Hence, we first verified that the molecular profiles observed in human cirrhosis were faithfully reproduced in 2 animal models of chronic liver injury. The DEN/CCl 4 chemically induced mouse model and the recently described nonalcoholic steatohepatitis/HCC model $^{26}$ reliably recapitulated the presence of a carcinogenic phenotype observed in liver tissues from patients belonging to the ICR.

To identify the most promising candidate therapies for novel chemopreventive strategies, we conducted an in silico analysis using a large compendium of gene sets $^{34}$ recapitulating the main targets of 1202 approved drugs. Among the top 10 most significantly enriched drugs, we selected nintedanib, the only therapy approved by the US Food and Drug Administration for non-neoplastic conditions. In the $\mathrm{DEN} / \mathrm{CCl}_{4}$ animal model, oral administration of nintedanib reduced the ICF, including the immunosuppressive ICF subtype, ultimately reducing HCC incidence and growth. Reversion of the ICF induced by treatment with nintedanib was accompanied by reduction of $\mathrm{CD}^{+}$lymphocytes, which could be due to its mechanism of action inhibiting the src family of kinases (ie, LCK, FLT3, and SRC). These findings are in line with previous reports suggesting that $\mathrm{CD}^{+}$cells propagate immune-mediated liver injury in models of chronic liver inflammation or autoimmune liver disease. ${ }^{43,44}$ Pretreatment with T-cell-specific antibodies or immunosuppressive agents, such as anti-CD4 monoclonal antibody, FK506 (tacrolimus), or cyclosporine A, have been shown to ameliorate hepatitis in these models, further supporting the role of $\mathrm{CD}^{+} \mathrm{T}$ cells in inducing liver damage. ${ }^{43}$ Results in a second animal model treated with the combination of the antiinflammatory drug, aspirin, and clopidogrel (a P2Y12 inhibitor) confirmed the therapeutic potential of immunomodulating the ICF and supported the pro-tumorigenic role exerted by the immune response. Indeed, only the treatment able to modulate the ICF, as indicated by the reduction of immune cells (ie, $\mathrm{CD}^{+}$and $\mathrm{NK} \mathrm{T}$ cells) and 
the reversion of the ICF signature, successfully reduced liver damage and prevented HCC development. Overall, our study identifies a novel promising chemopreventive strategy for HCC and confirms the validity of using the reversion of the ICF as reliable readout of efficacy. This is of great clinical importance because there is currently no effective method to monitor the short-term effects of chemopreventive drugs. ${ }^{5}$ Nintedanib belongs to a new generation of tyrosine kinase inhibitors that, in addition to exerting immune modulation, block the activation of main angiogenic receptors. ${ }^{45}$ Many cytokines and growth factors are involved in modulating the formation of new vessels. Expression of $V E G F$ and its receptors is elevated in HCC cell lines and tissues, as well as in the blood circulation of patients with HCC. ${ }^{33,46-48}$ In our model, nintedanib exerted its chemopreventive mechanisms in part through the inhibition of VEGF signaling, a major driver of angiogenesis. ${ }^{49}$ Thus far, independent studies had reported that HCC prevention can be achieved in animal models by attenuating liver fibrosis through the inhibition of $\mathrm{EGFR}^{50,51}$ or lysophosphatidic $\operatorname{acid}^{32}$ signaling. With the current study, we show that modulation of the liver microenvironment by molecular targeted drugs, which simultaneously block liver inflammation and angiogenesis, might represent a powerful alternative strategy.

We recently defined the immune class of $\mathrm{HCC}^{15}$ and the immune exclusion class (characterized by active Wnt/ CTNNB1), 3,15,52 which might predict response and primary resistance to checkpoint inhibitors, respectively., ${ }^{3,52}$ Here, we explored the immunomodulatory mechanisms underlying HCC occurrence by defining an immune-mediated field effect that conforms to a cancer-permissive milieu, thus placing these patients at the highest risk of HCC development. In addition, our preclinical data with a drug approved for pulmonology and non-small-cell lung cancer treatment suggest that the permissive microenvironment can be reverted, leading to a reduction in HCC occurrence. These data provide the rationale for testing this strategy in early chemoprevention trials targeting patients with cirrhosis who are at high risk of HCC development. In addition, this strategy could also be further explored in the adjuvant setting considering that $60 \%$ of HCC undergoing resection also present this permissive milieu in the adjacent nontumoral tissue.

\section{Supplementary Material}

Note: To access the supplementary material accompanying this article, visit the online version of Gastroenterology at www.gastrojournal.org, and at https://doi.org/10.1053/ j.gastro.2019.07.028.

\section{References}

1. Bray F, Ferlay J, Soerjomataram I, et al. Global cancer statistics 2018: GLOBOCAN estimates of incidence and mortality worldwide for 36 cancers in 185 countries. CA Cancer J Clin 2018;68:394-424.

2. Llovet JM, Zucman-Rossi J, Pikarsky E, et al. Hepatocellular carcinoma. Nat Rev Dis Prim 2016; 2:16018.

3. Llovet JM, Montal R, Sia D, et al. Molecular therapies and precision medicine for hepatocellular carcinoma. Nat Rev Clin Oncol 2018;15:599-616.

4. Galle PR, Forner A, Llovet JM, et al. EASL clinical practice guidelines: management of hepatocellular carcinoma. J Hepatol 2018;69:182-236.

5. Fujiwara N, Friedman SL, Goossens N, et al. Risk factors and prevention of hepatocellular carcinoma in the era of precision medicine. J Hepatol 2018;68:526-549.

6. Calvaruso V, Cabibbo G, Cacciola I, et al. Incidence of hepatocellular carcinoma in patients with HCVassociated cirrhosis treated with direct-acting antiviral agents. Gastroenterology 2018;155:411-421.

7. Kanwal F, Kramer J, Asch SM, et al. Risk of hepatocellular cancer in HCV patients treated with directacting antiviral agents. Gastroenterology 2017; 153:996-1005.

8. Lochhead P, Chan AT, Nishihara R, et al. Etiologic field effect: reappraisal of the field effect concept in cancer predisposition and progression. Mod Pathol 2015;28: 14-29.

9. Hoshida Y, Villanueva A, Kobayashi M, et al. Gene expression in fixed tissues and outcome in hepatocellular carcinoma. N Engl J Med 2008;359:1995-2004.

10. Hernandez-Gea V, Toffanin S, Friedman SL, et al. Role of the microenvironment in the pathogenesis and treatment of hepatocellular carcinoma. Gastroenterology 2013; 144:512-527.

11. Ji J, Eggert T, Budhu A, et al. Hepatic stellate cell and monocyte interaction contributes to poor prognosis in hepatocellular carcinoma. Hepatology 2015;62:481495.

12. Zhang DY, Goossens N, Guo J, et al. A hepatic stellate cell gene expression signature associated with outcomes in hepatitis C cirrhosis and hepatocellular carcinoma after curative resection. Gut 2016;65:1754-1764.

13. Kim JH, Sohn BH, Lee H-S, et al. Genomic predictors for recurrence patterns of hepatocellular carcinoma: model derivation and validation. PLoS Med 2014;11(12): e1001770.

14. Hoshida Y, Villanueva A, Sangiovanni A, et al. Prognostic gene expression signature for patients with hepatitis Crelated early-stage cirrhosis. Gastroenterology 2013; 144:1024-1030.

15. Sia D, Jiao $Y$, Martinez-Quetglas I, et al. Identification of an immune-specific class of hepatocellular carcinoma, based on molecular features. Gastroenterology 2017; 153:812-826.

16. Villanueva A, Portela A, Sayols S, et al. DNA methylation-based prognosis and epidrivers in hepatocellular carcinoma. Hepatology 2015;61:1945-1956.

17. Wang M, Gong Q, Zhang J, et al. Characterization of gene expression profiles in HBV-related liver fibrosis 
patients and identification of ITGBL1 as a key regulator of fibrogenesis. Sci Rep 2017;7:43446.

18. Godec J, Tan Y, Liberzon A, et al. Compendium of immune signatures identifies conserved and speciesspecific biology in response to inflammation. Immunity 2016;44:194-206.

19. Brunet J-P, Tamayo P, Golub TR, et al. Metagenes and molecular pattern discovery using matrix factorization. Proc Natl Acad Sci U S A 2004;101:41644169.

20. Newman AM, Liu CL, Green MR, et al. Robust enumeration of cell subsets from tissue expression profiles. Nat Methods 2015;12:453-457.

21. Bindea G, Mlecnik B, Tosolini M, et al. Spatiotemporal dynamics of intratumoral immune cells reveal the immune landscape in human cancer. Immunity 2013; 39:782-795.

22. Thorsson V, Gibbs DL, Brown SD, et al. The immune landscape of cancer. Immunity 2018;48:812-830.

23. Reich M, Liefeld T, Gould J, et al. GenePattern 2.0. Nat Genet 2006;38:500-501.

24. Charoentong $\mathbf{P}$, Finotello F, Angelova M, et al. Pancancer immunogenomic analyses reveal genotypeimmunophenotype relationships and predictors of response to checkpoint blockade. Cell Rep 2017; 18:248-262.

25. Dapito DH, Mencin A, Gwak G-Y, et al. Promotion of hepatocellular carcinoma by the intestinal microbiota and TLR4. Cancer Cell 2012;21:504-516.

26. Malehmir M, Pfister D, Gallage S, et al. Platelet GPIb $\alpha$ is a mediator and potential interventional target for NASH and subsequent liver cancer. Nat Med 2019; 25:641-655.

27. Okamoto M, Utsunomiya T, Wakiyama S, et al. Specific gene-expression profiles of noncancerous liver tissue predict the risk for multicentric occurrence of hepatocellular carcinoma in hepatitis C virus-positive patients. Ann Surg Oncol 2006;13:947-954.

28. Wolf DM, Lenburg ME, Yau C, et al. Gene coexpression modules as clinically relevant hallmarks of breast cancer diversity. PLoS One 2014;9(2): e88309.

29. Beck AH, Espinosa I, Edris B, et al. The macrophage colony-stimulating factor 1 response signature in breast carcinoma. Clin Cancer Res 2009;15:778-787.

30. Rooney MS, Shukla SA, Wu CJ, et al. Molecular and genetic properties of tumors associated with local immune cytolytic activity. Cell 2015;160:48-61.

31. Finkin S, Yuan D, Stein I, et al. Ectopic lymphoid structures function as microniches for tumor progenitor cells in hepatocellular carcinoma. Nat Immunol 2015; 16:1235-1244.

32. Nakagawa S, Wei L, Song WM, et al. Molecular liver cancer prevention in cirrhosis by organ transcriptome analysis and lysophosphatidic acid pathway inhibition. Cancer Cell 2016;30:879-890.

33. Chiang DY, Villanueva A, Hoshida Y, et al. Focal gains of VEGFA and molecular classification of hepatocellular carcinoma. Cancer Res 2008;68:6779-6788.
34. Yoo M, Shin J, Kim J, et al. DSigDB: drug signatures database for gene set analysis. Bioinformatics 2015; 31:3069-3071.

35. Richeldi L, du Bois RM, Raghu G, et al. Efficacy and safety of nintedanib in idiopathic pulmonary fibrosis. N Engl J Med 2014;370:2071-2082.

36. Wolf MJ, Adili A, Piotrowitz K, et al. Metabolic activation of intrahepatic $\mathrm{CD}^{+} \mathrm{T}$ cells and NKT cells causes nonalcoholic steatohepatitis and liver cancer via cross-talk with hepatocytes. Cancer Cell 2014;26: 549-564.

37. Brown ZJ, Heinrich B, Greten TF. Mouse models of hepatocellular carcinoma: an overview and highlights for immunotherapy research. Nat Rev Gastroenterol Hepatol 2018;15:536-554.

38. Coussens LM, Werb Z. Inflammation and cancer. Nature 2002;420(6917):860-867.

39. Hanahan D, Weinberg RA. Hallmarks of cancer: the next generation. Cell 2011;144:646-674.

40. Makarova-Rusher OV, Medina-Echeverz J, Duffy AG, et al. The yin and yang of evasion and immune activation in HCC. J Hepatol 2015;62:1420-1429.

41. Papatheodoridis GV, Dalekos GN, Yurdaydin C, et al. Incidence and predictors of hepatocellular carcinoma in Caucasian chronic hepatitis B patients receiving entecavir or tenofovir. J Hepatol 2015; 62:363-370.

42. Morgan RL, Baack B, Smith BD, et al. Eradication of hepatitis $\mathrm{C}$ virus infection and the development of hepatocellular carcinoma: a meta-analysis of observational studies. Ann Intern Med 2013;158:329-337.

43. Tiegs G, Hentschel J, Wendel A. A T cell-dependent experimental liver injury in mice inducible by concanavalin A. J Clin Invest 1992;90:196-203.

44. Omenetti S, Brogi M, Goodman WA, et al. Dysregulated intrahepatic $\mathrm{CD}^{+}{ }^{+}$T-cell activation drives liver inflammation in ileitis-prone SAMP1/YitFc mice. Cell Mol Gastroenterol Hepatol 2015;1:406-419.

45. Hilberg F, Roth GJ, Krssak M, et al. BIBF 1120: triple angiokinase inhibitor with sustained receptor blockade and good antitumor efficacy. Cancer Res 2008; 68:4774-4782.

46. Mas VR, Maluf DG, Archer KJ, et al. Angiogenesis soluble factors as hepatocellular carcinoma noninvasive markers for monitoring hepatitis $\mathrm{C}$ virus cirrhotic patients awaiting liver transplantation. Transplantation 2007; 84:1262-1271.

47. Poon RTP, Ho JWY, Tong CSW, et al. Prognostic significance of serum vascular endothelial growth factor and endostatin in patients with hepatocellular carcinoma. Br J Surg 2004;91:1354-1360.

48. Horwitz E, Stein I, Andreozzi M, et al. Human and mouse VEGFA-amplified hepatocellular carcinomas are highly sensitive to sorafenib treatment. Cancer Discov 2014; 4:730-743.

49. Carmeliet P. VEGF as a key mediator of angiogenesis in cancer. Oncology 2005;69(Suppl 3):4-10.

50. Schiffer E, Housset C, Cacheux W, et al. Gefitinib, an EGFR inhibitor, prevents hepatocellular carcinoma 
development in the rat liver with cirrhosis. Hepatology 2005;41:307-314.

51. Fuchs BC, Hoshida Y, Fujii T, et al. Epidermal growth factor receptor inhibition attenuates liver fibrosis and development of hepatocellular carcinoma. Hepatology 2014;59:1577-1590.

52. Pinyol R, Sia D, Llovet JM. Immune exclusion-Wnt/ CTNNB1 class predicts resistance to immunotherapies in HCC. Clin Cancer Res 2019;25:2021-2023.

\section{Author names in bold designate shared co-first authorship.}

\section{Received January 28, 2019. Accepted July 17, 2019.}

\section{Reprint requests}

Address requests for reprints to Josep M. Llovet, MD, Liver Cancer Translational Research Laboratory, IDIBAPS-Hospital Clinic, Rosselló 153, 08039, Barcelona, Catalonia, Spain. e-mail: jmllovet@clinic.cat; fax: +34933129406; or Daniela Sia, PhD, Mount Sinai Liver Cancer Program, Division of Liver Diseases, Tisch Cancer Institute, Icahn School of Medicine at Mount Sinai, Madison Ave 1425. 11F-70. Box 1123, New York, NY 10029. e-mail: daniela.sia@mssm.edu; fax: +1212.849.2574.

\section{Acknowledgements}

The authors thank Juan José Lozano for technical assistance in the normalization of the transcriptomic array of the animal model. This study was developed at the building of Centre Esther Koplowitz from IDIBAPS/CERCA Programme/Generalitat de Catalunya. The authors also acknowledge Angelo Sangiovanni and Massimo Colombo for providing the seminal cohort of patients with cirrhotic in our previous studies. ${ }^{12,14}$

Author contributions: Study concept and design: Agrin Moeini, Victoria Tovar, Daniela Sia, and Josep M. Llovet; acquisition of data: Agrin Moeini, Sara Torrecilla, Carla Montironi, Judit Peix, Mónica Higuera, and Manel Sole; analysis and interpretation of data: Agrin Moeini, Sara Torrecilla, Victoria Tovar, Carla Montironi, Carmen Andreu-Oller, and Manel Sole; drafting of the manuscript: Agrin Moeini, Daniela Sia, and Josep M. Llovet; critical revision of the manuscript for important intellectual content: Roser Pinyol, Scott L. Friedman, Daniela Sia, and Josep M. Llovet; obtained funding: Josep M. Llovet; study supervision: Josep M. Llovet and Daniela Sia.

\section{Conflicts of interest}

These authors disclose the following: Part of the study was supported with an investigator-initiated research grant by Boehringer Ingelheim. Josep M. Llovet has been a consultant and advisory board member and has received research funding from Boehringer Ingelheim and is receiving research support from Bayer HealthCare Pharmaceuticals, Eisai Inc, Bristol-Myers Squibb, and Ipsen and consulting fees from Bayer HealthCare Pharmaceuticals, BristolMyers Squibb, Eisai Inc, Celsion Corporation, Eli Lilly, Exelixis, Merck, Ipsen, Glycotest, Navigant, Leerink Swann LLC, Midatech Ltd, Fortress Biotech, Sprink Pharmaceuticals, and Nucleix. Scott L. Friedman has been a consultant for Abide Therapeutics, Allergan Pharmaceuticals, Angion Biomedica, Blade Therapeutics, Can-Fite Biopharma, Enanta Pharmaceuticals, Escient Therapeutics, Forbion, Galmed, Genfit, Glycotest, Glympse Bio, Metacrine Inc, Mistral Biosciences, Morphic Rock Therapeutics, North Sea Therapeutics, Novartis, Novo Nordisk, Pfizer Pharmaceuticals, Salix Pharmaceuticals, Scholar Rock, Seal Rock Therapeutics, Second Genome, Surrozen, Symic Bio, Viking Therapeutics, and Kintai; has received research funding from Blade Therapeutics, Can-Fite Biopharma, Ferring Research Institute, Galmed; and has stock options from Intercept, Exalenz, Madrigal, Akarna Therapeutics, BirdRock Bio, Blade Therapeutics, Conatus, DeuteRx, Exalenz, Galectin, Galmed, Genfit, Glympse. The remaining authors disclose no conflicts.

\section{Funding}

Josep M. Llovet is supported by the European Commission (EC)/Horizon 2020 Program (HEPCAR, reference no. 667273-2); US Department of Defense (CA150272P3); an Accelerator Award (CRUCK, AECC, AIRC) (HUNTER, reference no. C9380/A26813), NCl Cancer Center Support Grant, National Cancer Institute; Tisch Cancer Institute (P30-CA196521); Samuel Waxman Cancer Research Foundation; Spanish National Health Institute (SAF201676390); and the Generalitat de Catalunya/AGAUR (SGR-1358). Agrin Moeini is supported by Spanish National Health Institute. Sara Torrecilla and Judit Peix are funded by Centro de Investigación Biomedica en Red de Enfermedades Hepáticas y Digestivas (Ciberehd-ISCIII). Carla Montironi is a recipient of Josep Font grant. Carmen Andreu-Oller is supported by "la Caixa" INPhINIT Fellowship Grant (LCF/BQ/IN17/11620024). Roser Pinyol is supported by HEPCAR and AECC. Daniela Sia is supported by the Gilead Sciences Research Scholar Program in Liver Disease. Scott L. Friedman is supported by the National Institutes of Health Research project grant (R01,DK5662) and US Department of Defense (CA150272P3). Mathias Heikenwälder was supported by an ERC Consolidator grant (HepatoMetaboPath), the SFBTR 209, 1335 and SFBTR179. This project has received funding from the European Union's Horizon 2020 research and innovation program under grant agreement no. 667273 and the Helmholtz future topic (Zukunftsthema) Immunology and Inflammation. 


\section{Supplementary Methods}

\section{Histologic Analysis of Human Samples}

Histologic evaluation was performed in 98 tissue samples obtained from patients with early HCC included in the study cohort. The portal/septal, pericentral, and lobular inflammation were assessed as follows: 0 , absent; 1 , mild; 2 , moderate; and 3 , marked. The presence or absence (1 or 0 , respectively) of interface inflammation and lymphoid aggregates was also evaluated. The latter structures were mainly found in the periportal/periseptal areas. An inflammatory infiltrate score was created by summing the values given by the portal/septal, lobular, and interface inflammation. Pericentral inflammation was not considered for the scoring system because $57 \%$ of patients had cirrhosis. According to the final score, we defined 2 inflammatory categories: the absent/mild category if the score was $<3$ and the moderate/marked category if the score was $\geq 3$. The presence or absence of cirrhosis was defined according to the METAVIR algorithm ${ }^{1}$ (F0-1-2-3/ F4). Ductular proliferation was also considered. Steatosis was assessed based on the size of the fat vesicles (macrovesicular or microvesicular) and the localization of the fat droplets in the liver parenchyma (periseptal/periportal, pericentral, or lobular). The presence or absence of ballooning, apoptotic bodies, and oncocytic change was also evaluated. Immunohistochemistry for CD4 and CD8 was performed in a subset of 70 patients of the training cohort. Staining was carried out on $3-\mu \mathrm{m}$-thick formalin-fixed paraffin-embedded tissue sections collected from a $40^{\circ}-$ $44^{\circ} \mathrm{C}$ flotation bath containing deionized water and mounted on $25 \times 75 \mathrm{~mm}$ positively $(+)$ charged slides. The slides were dried at $60^{\circ} \mathrm{C}$ in a convection oven for 30 minutes. Deparaffinization was performed, followed by standard cell conditioning 1 (ULTRA CC1 from Ventana Medical Systems, Tucson, AZ). Staining was performed with the Ventana BenchMark ULTRA system with the primary antibody for approximately 20 minutes at $36^{\circ} \mathrm{C}$. Signal was captured using the Ultraview Universal diaminobenzidine detection kit and blocked with antibody diluent (REF 251-018) for 8 minutes. Samples were counterstained with hematoxylin for 4 minutes and post-counterstained with Bluing Reagent for 4 minutes. The primary antibodies used were anti-CD4 (clone SP35; Roche, Basel, Switzerland) and anti-CD8 (clone SP57, Roche). The quantification was done automatically (Image J, National Institutes of Health, Bethesda, MD) by calculating the positive areas $\left(\mu \mathrm{m}^{2}\right)$-considering 5 random areas within the lobular and portal/septal infiltrates of the nontumor liver tissue-at 200 times magnification and using the same threshold for all samples.

\section{Generation of the Diethylnitrosamine/Carbon Tetrachloride Animal Model}

The chemically induced (DEN/CCl 4$)$ model was generated as previously described. ${ }^{2,3}$ HCC was induced by a single intraperitoneal injection of DEN (Sigma-Aldrich, St. Louis, MO; $25 \mathrm{mg} / \mathrm{kg}$ intraperitoneally, dissolved in $0.9 \%$ sodium chloride solution) given at day 15 postpartum followed by $11-14$ weekly injections of $\mathrm{CCl}_{4}(0.5 \mathrm{~mL} / \mathrm{kg}$ intraperitoneally, dissolved in corn oil) starting at 4 weeks of age (Supplementary Figure $7 A$ ). Mice $(\mathrm{N}=55)$ were randomly assigned at 12 weeks of age to receive $50 \mathrm{mg} / \mathrm{kg} /$ day of nintedanib (Boehringer Ingelheim) $(n=29)$ or vehicle $(n=26)$. The vehicle solution was formulated as follows: $1.8 \%$ hydroxypropyl $\beta$-cyclodextrin, $5 \%$ acetic acid (10\%), and Natrosol (0.5\%) (Sigma-Aldrich (\#54290), Saint Louis, M0). At 15, 17, and 18 weeks of age, mice were killed 48 hours after the last dose of $\mathrm{CCl}_{4}$, having been treated with nintedanib for 3,5 , and 6 weeks, respectively $(n=15-21$ per time point). Immediately after mice were killed, livers were explanted, digitally photographed, and weighed. The evaluation of macroscopic malignant nodules was assessed by 2 independent investigators. Based on visual criteria, a hepatic lesion with a diameter $>0.5 \mathrm{~mm}$ and with dysmorphic and/or dyschromic surface was considered a hepatic tumor. The diameter of tumors was measured with a hand caliper. The biological endpoints for chemopreventive efficacy were (1) incidence of hepatic tumors; (2) number of tumors; and (3) size, considering the largest diameter of all counted tumors. The largest liver lobe was fixed in buffered $4 \%$ paraformaldehyde for 24 hours for posterior histologic and immunohistochemical analysis. In addition, samples of adjacent nontumor liver were snap frozen at $-80^{\circ} \mathrm{C}$ for subsequent RNA and protein analysis. Potential treatmentrelated toxicity was evaluated by monitoring body weight losses and quantitative determination of serum aspartate aminotransferase and alanine aminotransferase.

\section{Histologic and Immunohistochemical Analysis of the Mouse Samples}

FFPE mouse liver samples were cut in $4-\mu \mathrm{m}$ sections and stained with H\&E for further histologic examination. The samples were assessed, with nontumor and tumor tissue considered, by 2 expert liver pathologists (CM, MS) blinded to the treatments. In the nontumor tissue, the number of lymphoid aggregates was determined. These were defined as polarized aggregates composed mainly of lymphocytes with scant plasma cells, neutrophils, and macrophages localized in the periportal and pericentral areas with a measure no bigger than $0.1 \mathrm{~mm}$. The presence of inflammatory infiltrates was also determined for the periportal, pericentral, and lobular areas using a scoring system as follows: 0 , absence or rare; 1 , mild; 2 , mild/ moderate; 3, moderate; 4, moderate/marked; and 5, marked. Other variables, such as the presence of microabscesses, ductular proliferation $(0$, absence; $1,<25 \%$ of portal tracts involved; 2, 25\%-50\% of portal tracts involved; $3,>50 \%$ of portal tracts involved), necrosis, steatosis, and apoptotic bodies, were also determined. Ballooning was defined according to the following scale: 0 , absence; 1 , focal; 2 , multiple foci; 3 , diffuse. Hepatic fibrosis was assessed by Sirius Red staining. The METAVIR algorithm $^{1}$ was used to grade hepatic fibrosis from F0 (no scarring) to F4 (cirrhosis or severe fibrosis). Also, perisinusoidal fibrosis was evaluated as follows: $1,0 \%-5 \% ; 2$, 
$5 \%-33 \% ; 3,33 \%-66 \%$, and $4,>60 \%$. Histologically, hepatic tumors presented a high cellular density composed of small cells with altered nuclear/cytoplasmic ratio and a clear cytoplasm. Among these hepatic tumors, those that were circular, well-defined lesions with pushing margins were diagnosed as HCCs, whereas a dysplastic nodule was considered when the lesion had an ill-defined shape within the liver parenchyma. The presence of vascular invasion was a characteristic attributed to HCC. The size of both lesions was not used to make a distinction between them, although HCCs tend to be larger than dysplastic nodules. Finally, for the evaluation of the chemopreventive effects of a tyrosine kinase inhibitor in adjacent liver tissue, FFPE sections from mice killed at 17 weeks of age were analyzed by immunohistochemistry ( $\mathrm{n}=5$ mice per treatment arm). Heat-induced antigen retrieval was performed with 10 mmol/L sodium citrate buffer $(\mathrm{pH} 6.0)$ or $0.5 \mathrm{~mol} / \mathrm{L}$ Tris buffer ( $\mathrm{pH} 10.0$ ) for 15 minutes (5 minutes, 3 times), and the reaction was quenched using hydrogen peroxide $3 \%$. After washing with phosphate-buffered saline, samples were incubated with anti-pERK (phosphoThr202/Tyr204) (Cell Signaling, Danvers, MA), anti-CD31 (Abcam, Cambridge, UK), and anti-CD4 and anti-CD8 (both from Cell Signaling). Diaminobenzidine (3,3'-diaminobenzidine) was used as detection system (EnVision+ System-HRP; Dako, Santa Clara, CA). Morphometric quantification of the percent area of liver vasculature $(n=5$ mice per treatment arm) was performed by evaluating the mean area of CD31 staining in randomly selected low-magnification fields $(\mathrm{n}=$ $5,10 \times$ fields for CD31 staining; $n=10,20 \times$ fields for collagen) with ImageJ software.

\section{RNA Extraction and Gene Expression Profiling of Mice Livers}

Total RNA from the 2 mouse models described was extracted from $20 \mathrm{mg}$ fresh-frozen nontumoral liver tissue with Trizol reagent (Invitrogen, Waltham, MA) and purified with RNeasy columns (Qiagen, Valencia, CA). RNA sample concentration and quality were assessed by NanoDrop ND1000 spectrometer (NanoDrop, Wilmington, DE) and bioanalyzer (Agilent, Palo Alto, CA), respectively. Gene expression microarray studies were conducted using the Gene Chip HT MG-430 (Affymetrix, Santa Clara, CA). The raw .cel files were background corrected and normalized with the robust multiarray averaging procedure, ${ }^{4}$ with a custom chip definition file (.cdf) from the Custom CDF project (HTMG430PM_Mm_ENTREZG v18.0). ${ }^{5}$ To assess ICF-signature enrichment through ssGSEA (GenePattern), mice genes were humanized, and intensity values were log transformed. For quantitative reverse-transcription polymerase chain reaction analysis, complementary DNA was synthesized from $1 \mu \mathrm{g}$ purified total RNA using SuperScript III reverse transcriptase (Invitrogen). Relative gene expression levels were measured by TaqMan Gene Expression Assays (Applied Biosystems, Foster City, CA) by using specific probes for Col1a1 (Mm00801666_g1), Col1a2 (Mm00483888_m1), Acta2 (Mm01546133_m1), and Pdgfrb (Mm00435546_m1). The housekeeping gene GAPDH (Mm99999915_g1) was used for normalization. Microarray data of these newly profiled samples are in the Gene Expression Omnibus under accession number GSE125975.

\section{Western Blot Analysis}

Whole-cell extracts were collected in lysis buffer $(50$ mmol/L Tris $\mathrm{pH} \mathrm{7.4,} 150 \mathrm{mmol} / \mathrm{L} \mathrm{NaCl}, 1 \%$ Triton X-100, $0.1 \%$ sodium dodecyl sulfate, $0.25 \mathrm{mmol} / \mathrm{L}$ EDTA, $1 \%$ sodium deoxycholate) containing phosphatases and proteases inhibitors (Roche). Next, 30-70 $\mu \mathrm{g}$ of total protein were resolved in polyacrylamide gels and transferred to nitrocellulose membranes (Pierce, Rockford, IL). Membranes were blocked with bovine serum albumin and hybridized overnight at $4^{\circ} \mathrm{C}$ with primary antibodies against VEGFR-2, pVEGFR-2 (Tyr951), Akt, pAkt (Ser473), ERK1/2, pERK1/2 (Thr202/Tyr204), Bcl-xL, PARP (all from Cell Signaling), and tubulin (Sigma-Aldrich). Appropriate horseradish peroxidase-conjugated secondary antibodies (Dako) were incubated for 1 hour at room temperature, and immunoreactivity was detected with a LAS-3000 imaging system (Fujifilm, Tokyo, Japan) by using Amersham ECL Prime Western Blotting Detection Reagent (GE Healthcare, Chicago, IL). 


\section{Supplementary References}

1. Bedossa P, Poynard T. An algorithm for the grading of activity in chronic hepatitis C. The METAVIR Cooperative Study Group. Hepatology 1996;24:289-293.

2. Uehara T, Ainslie GR, Kutanzi K, et al. Molecular mechanisms of fibrosis-associated promotion of liver carcinogenesis. Toxicol Sci 2013;132:53-63.

3. Dapito DH, Mencin A, Gwak G-Y, et al. Promotion of hepatocellular carcinoma by the intestinal microbiota and TLR4. Cancer Cell 2012;21:504-516.

4. Irizarry RA, Hobbs B, Collin F, et al. Exploration, normalization, and summaries of high density oligonucleotide array probe level data. Biostatistics 2003;4:249264.

5. Dai M, Wang P, Boyd AD, et al. Evolving gene/ transcript definitions significantly alter the interpretation of GeneChip data. Nucleic Acids Res 2005;33(20): e175.
6. Nakagawa S, Wei L, Song WM, et al. Molecular liver cancer prevention in cirrhosis by organ transcriptome analysis and lysophosphatidic acid pathway inhibition. Cancer Cell 2016;30:879-890.

7. Hoshida Y, Villanueva A, Kobayashi M, et al. Gene expression in fixed tissues and outcome in hepatocellular carcinoma. N Engl J Med 2008;359:1995-2004.

8. Kim JH, Sohn BH, Lee H-S, et al. Genomic predictors for recurrence patterns of hepatocellular carcinoma: model derivation and validation. PLoS Med 2014;11(12): e1001770.

9. Ji J, Eggert T, Budhu A, et al. Hepatic stellate cell and monocyte interaction contributes to poor prognosis in hepatocellular carcinoma. Hepatology 2015;62:481-495.

10. Zhang DY, Goossens N, Guo J, et al. A hepatic stellate cell gene expression signature associated with outcomes in hepatitis C cirrhosis and hepatocellular carcinoma after curative resection. Gut 2016;65:17541764. 
A

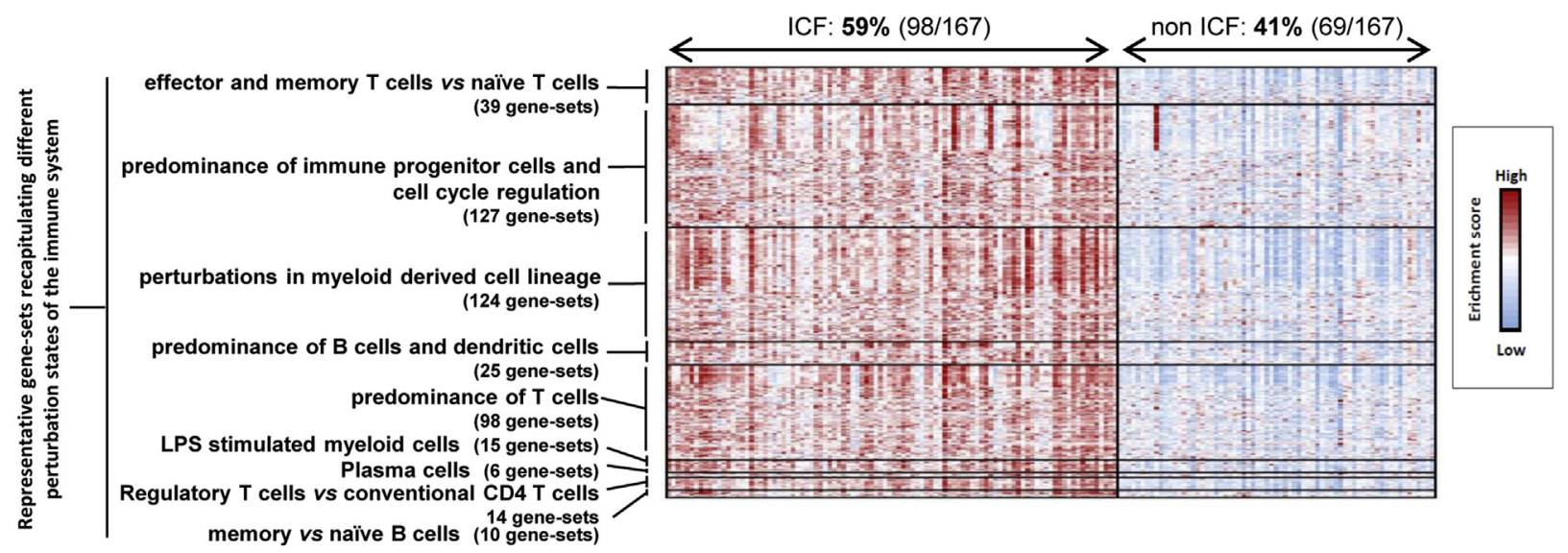

B
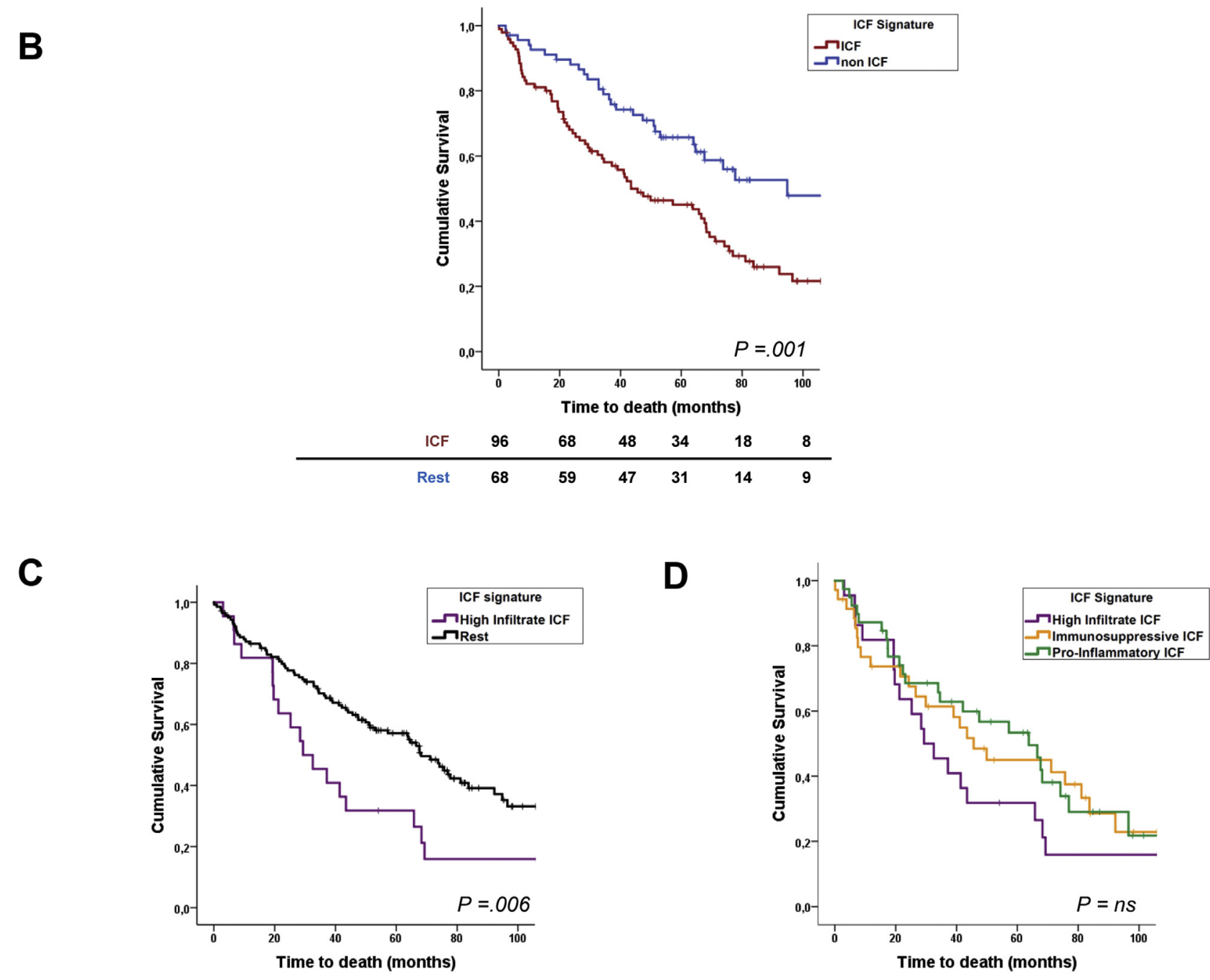

\begin{tabular}{rrrrrrr} 
High Infiltrate ICF & 22 & 15 & 9 & 6 & 3 & 3 \\
\hline Rest & 142 & 112 & 86 & 59 & 29 & 14
\end{tabular}

\begin{tabular}{rcccccc} 
High Infiltrate ICF & 22 & 15 & 9 & 6 & 3 & 3 \\
\hline Immunosuppressive ICF & 35 & 24 & 18 & 12 & 9 & 3 \\
\hline Pro-Inflammatory ICF & 39 & 29 & 21 & 16 & 6 & 2
\end{tabular}

Supplementary Figure 1. Identification of an ICF in the nontumor liver tissues and its association with overall survival in patients with early HCC. (A) Consensus-clustered analysis of gene sets recapitulating the different cell states and perturbations within the immune system. The figure includes a subgroup of representative gene sets among the approximately 5000 gene sets evaluated (Collection C7 of MsigDB). (B-D) Kaplan-Meier estimates of overall survival according to $(B)$ the presence of the ICF, $(C)$ the presence of the high-infiltrate subtype, and $(C)$ the different ICF subtypes. ns, not significant. 
A

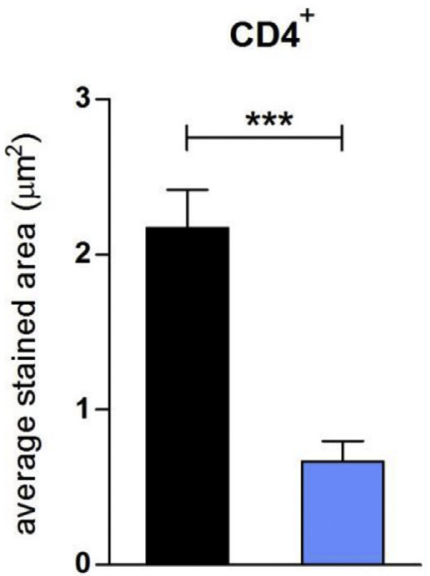

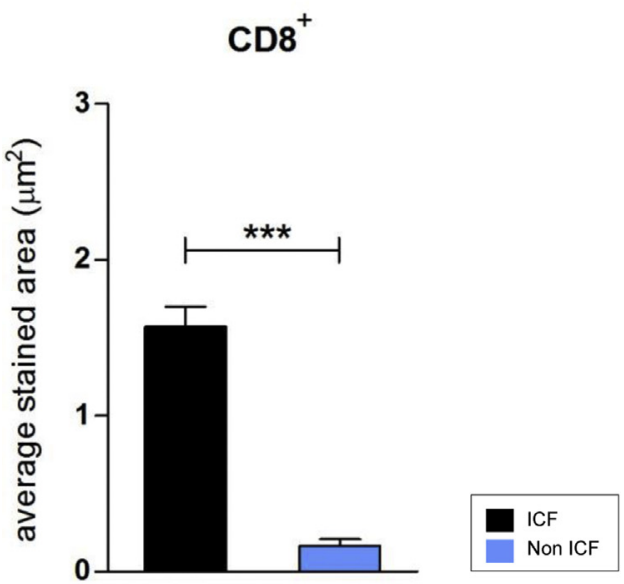

B

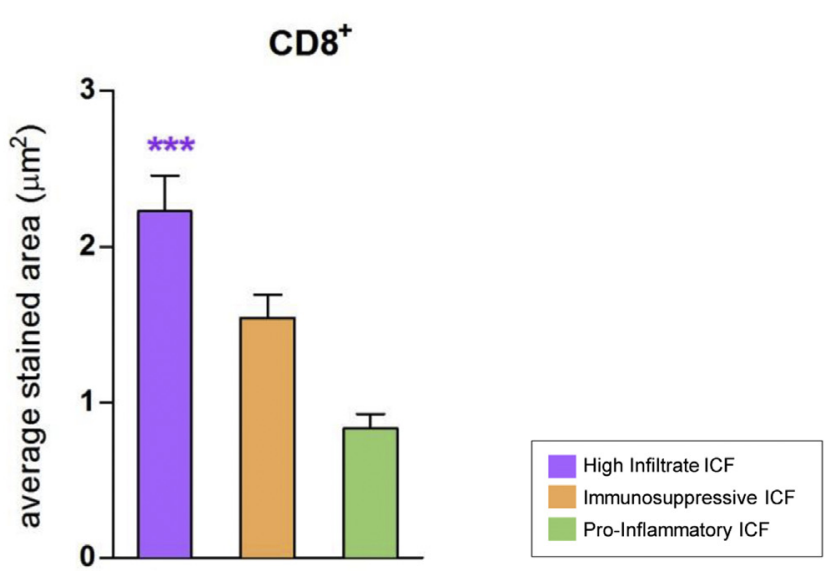

Supplementary Figure 2. Immunohistochemistry assessment of CD4 and CD8 positivity in nontumor liver tissues. Average stained area was automatically quantified and compared in $(A)$ samples from patients belonging to the ICF and non-ICF subgroups, and $(B)$ between ICF subtypes. $\mathrm{n}=15-19$ samples per group. ${ }^{* \star \star} P<.001$.

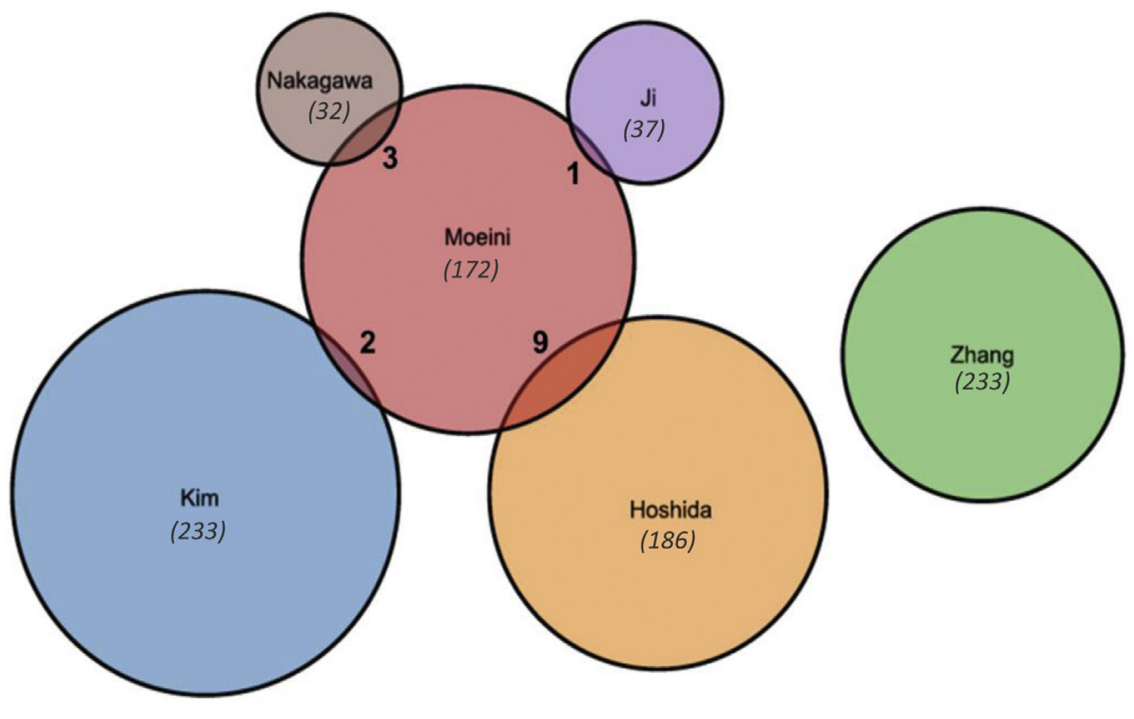

Supplementary Figure 3. Gene overlap between the 172 ICF gene-expression signature and other previously reported poor-prognosis expression signatures in nontumor liver tissue. Signatures are denoted by the name of the first author of each of the publications. ${ }^{6-10}$ Numbers in brackets indicate the total number of genes that constitute each signature, and bold numbers represent the number of genes that overlap between each signature and the ICF signature (here, referred to as Moeini). 
A

Validation cohort (GSE10143, N = 225 non-tumor liver tissues)

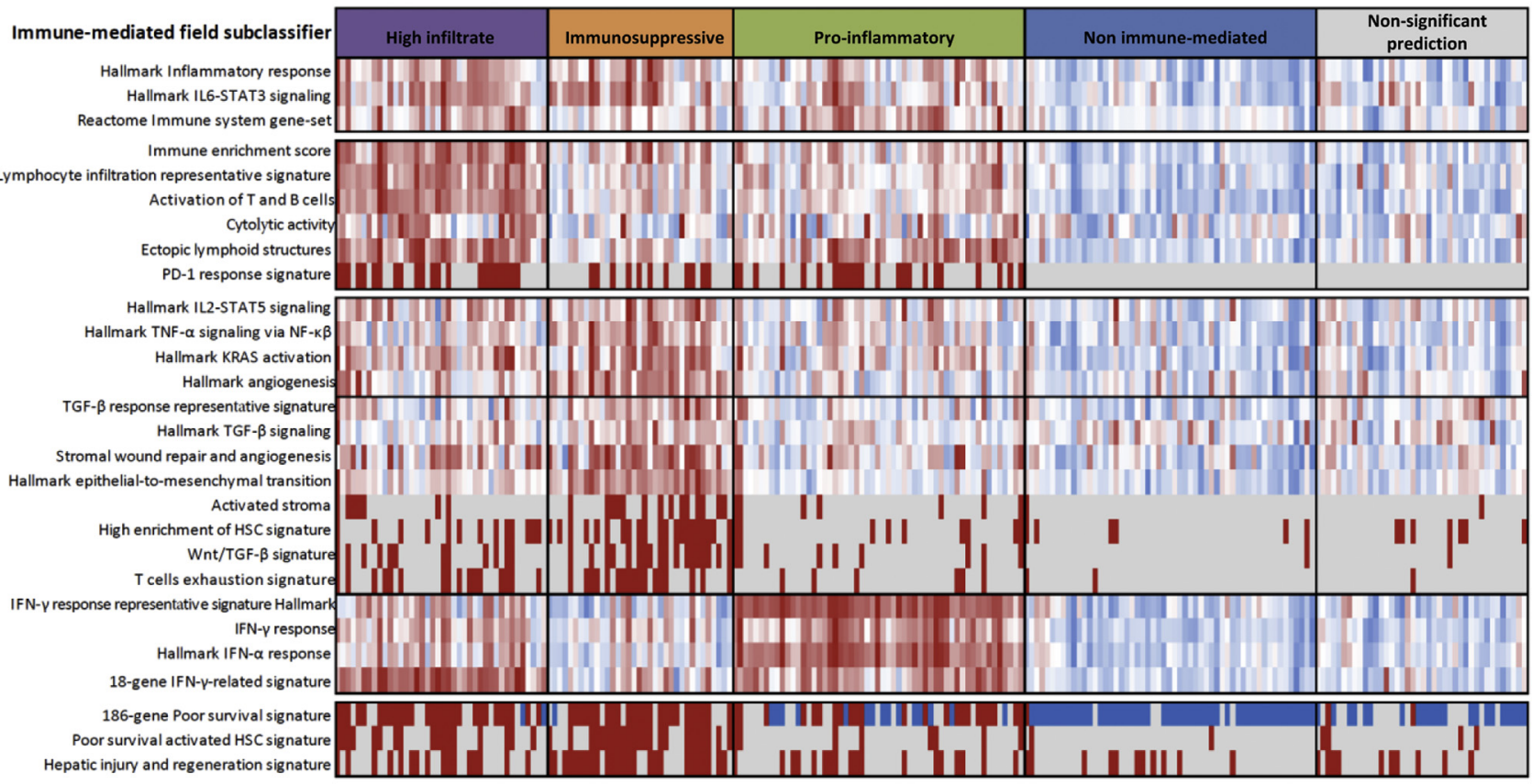

\begin{tabular}{|c|c|c|c|c|}
\hline \multirow{2}{*}{\multicolumn{2}{|c|}{$\frac{\text { SsGSEA }}{\text { Enrichment score }}$}} & \multirow{2}{*}{$\begin{array}{c}\text { NTP prediction } \\
\text { Presence of signature }\end{array}$} & \multicolumn{2}{|c|}{ 186-gene prognostic signature } \\
\hline & & & Poor survival & non-significant \\
\hline Low & High & Absence of signature & Good survival & \\
\hline
\end{tabular}

B

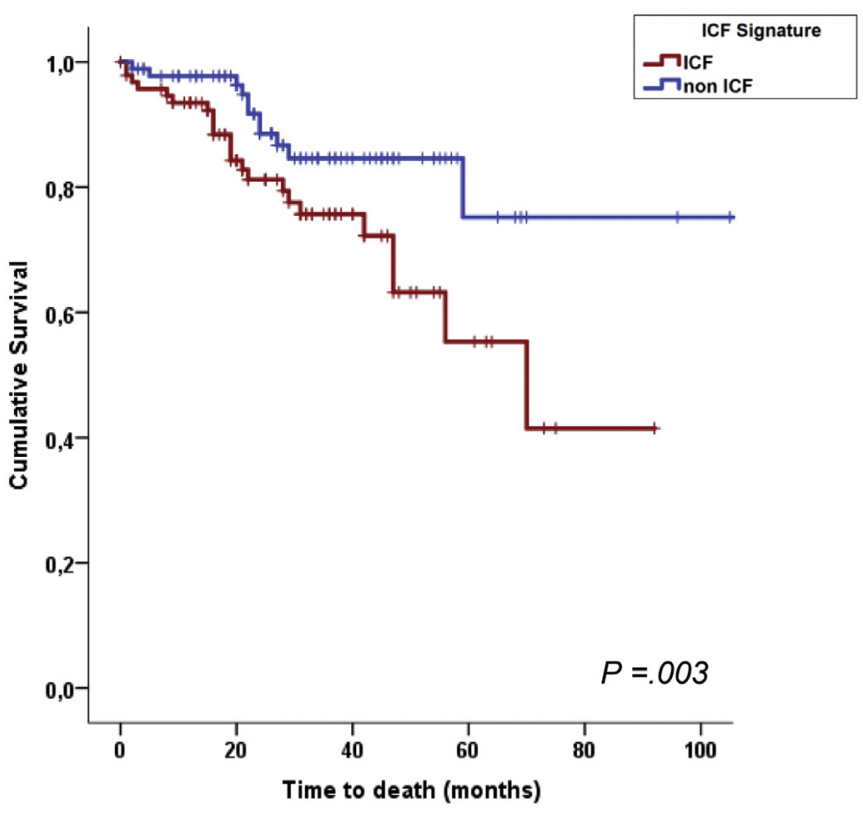

\begin{tabular}{ccccccc} 
ICF & 94 & 57 & 22 & 7 & 1 & 0 \\
\hline Non ICF & 90 & 64 & 25 & 8 & 4 & 3
\end{tabular}

Supplementary Figure 4. External validation of the molecular and clinical features of the ICF and its subtypes. (A) The main molecular characteristics of the ICF and the 3 distinct molecular subtypes were validated in a previously reported cohort that included 225 FFPE nontumor liver tissue samples from patients with early HCC profiled by CDNA-mediated Annealing, Selection, Extension, and Ligation (DASL) array. (B) Kaplan-Meier estimates of overall survival according to the presence of the ICF. NTP, nearest template prediction. 
A
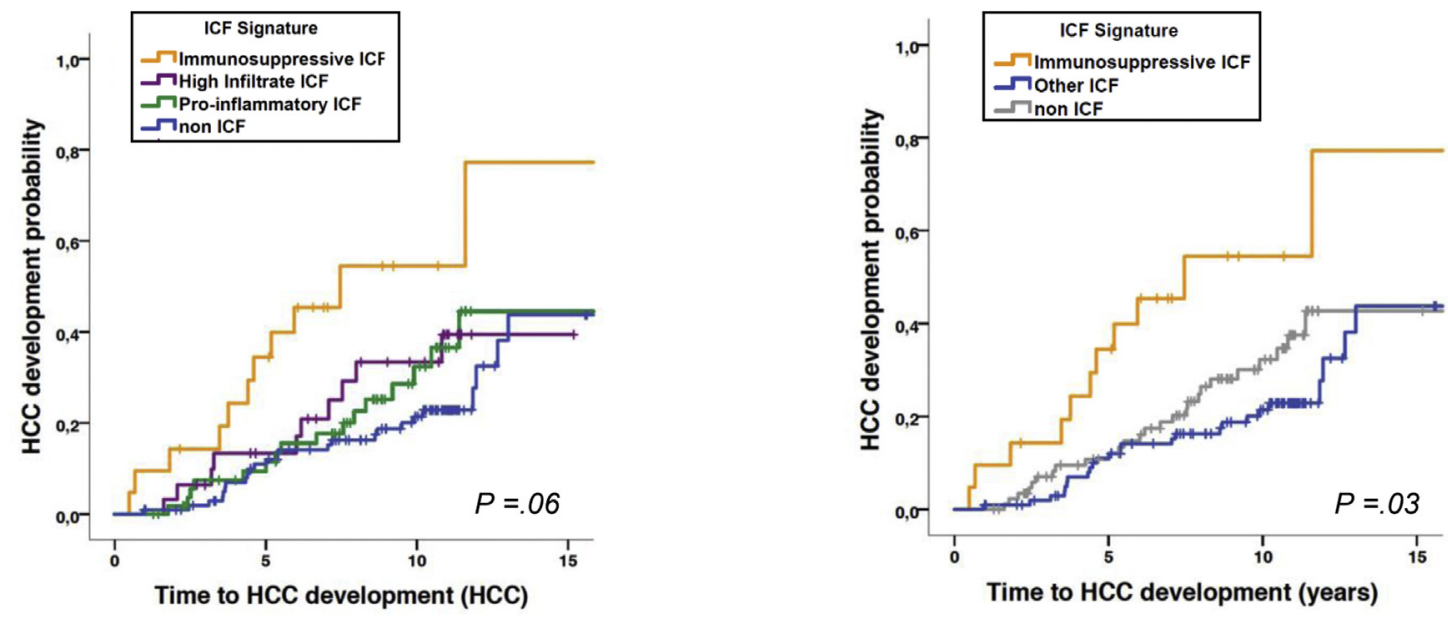

\begin{tabular}{rcccc} 
Immunosuppressive ICF & 21 & 13 & 3 & 1 \\
\hline High Infiltrate ICF & 31 & 23 & 13 & 1 \\
\hline Pro-Inflammatory ICF & 58 & 45 & 18 & 3 \\
\hline Non ICF & 106 & 87 & 57 & 10
\end{tabular}

\begin{tabular}{rcccc} 
Immunosuppressive ICF & 21 & 13 & 3 & 1 \\
\hline Other ICF & 89 & 68 & 31 & 3 \\
\hline Non ICF & 106 & 87 & 57 & 10
\end{tabular}

B
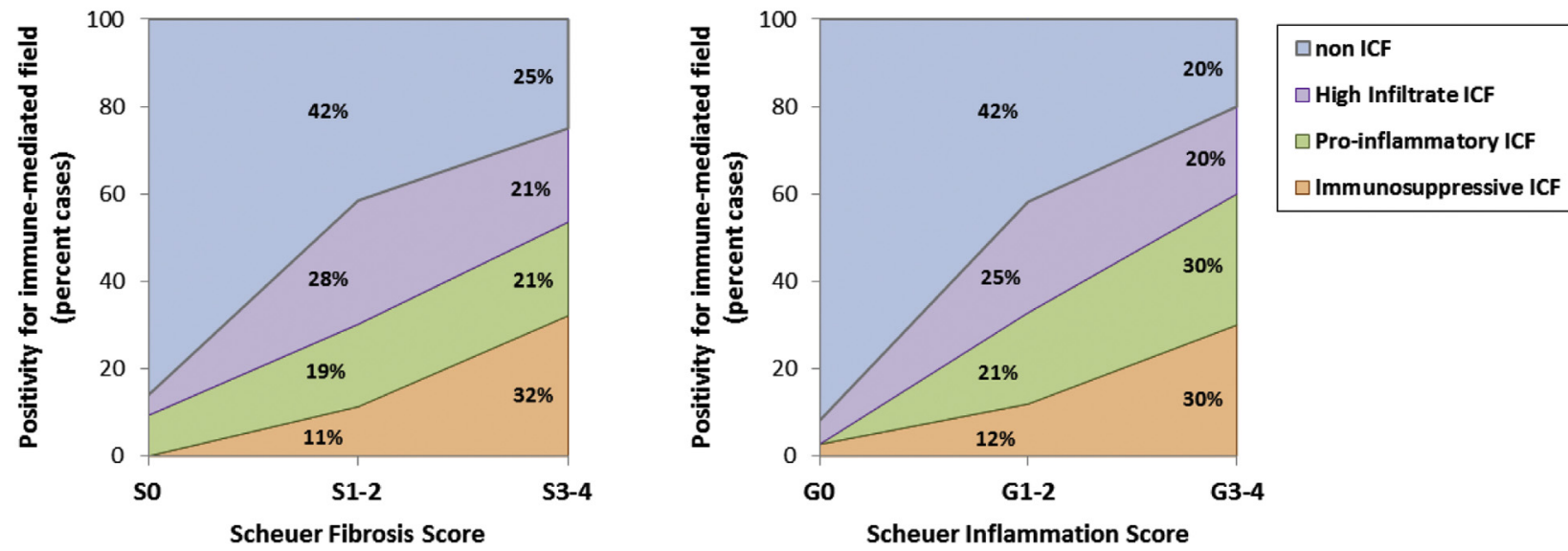

Supplementary Figure 5. Association of the presence of ICF subtypes with risk of HCC development and advanced liver disease in patients with fibrosis. (A) Kaplan-Meier analysis of the correlation of the ICF subtypes with risk of HCC development. (B) Correlation of the prediction of ICF subtypes with fibrosis and inflammation degree. Fibrosis degree was classified as none (S0), low (S1/S2), and high (S3/S4). Inflammation degree was classified as none (G0), low (G1/G2), and high (G3/G4). Non ICF, non-ICF subtype and unclassified cases; other ICF: high-infiltrate and proinflammatory ICFs. 
A

\section{Supplementary}

Figure 6. In silico enrichment analysis of gene sets recapitulating the targets of approved molecular therapies in nontumor liver of patients with early HCC. (A) Heatmap representing the enrichment scores of gene sets recapitulating the molecular targets of top enriched therapies in nontumor liver of HCC patients harboring the ICF compared with non-ICF/ other. (B) Constellation map representation of enrichment of the gene set of the main targets of topscored therapies centered around the ICF phenotype. The blue lines denote presence of overlapping genes among the different gene sets. AUC.pval, area under the curve $P$ value; NMI, normalized mutual information; t.pval, $t$ statistic $P$ value; t.stat, $t$ statistic.

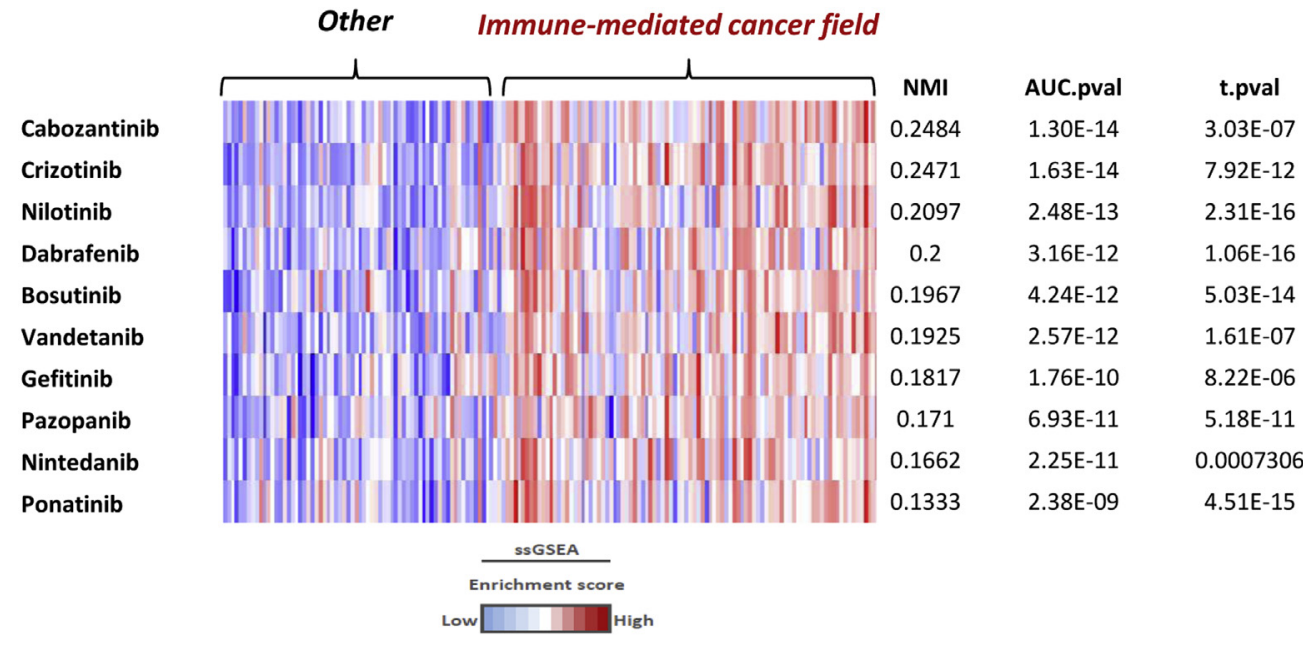

B

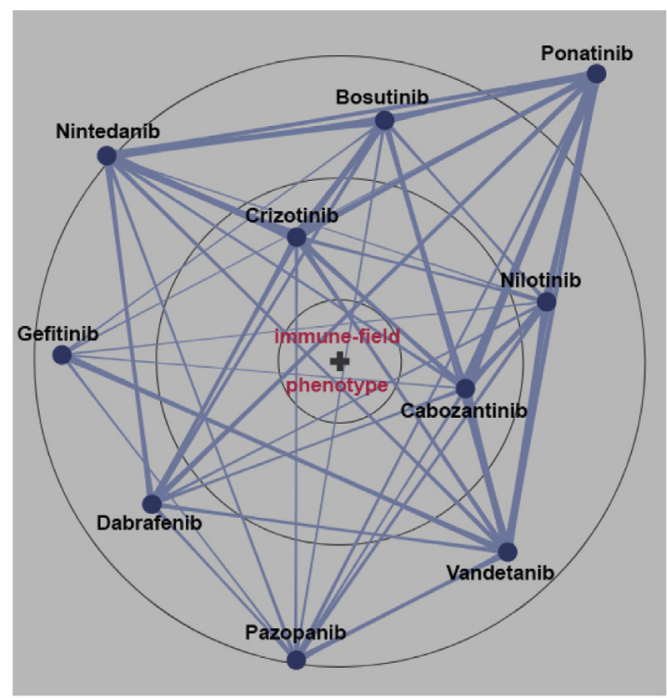


A

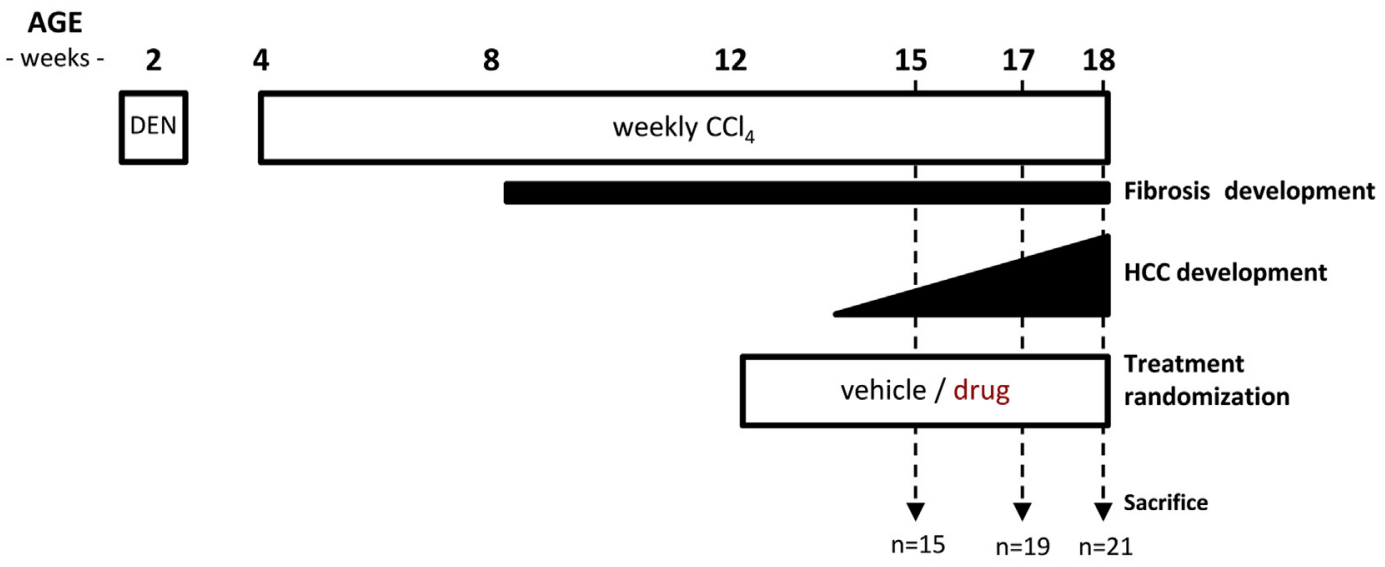

B

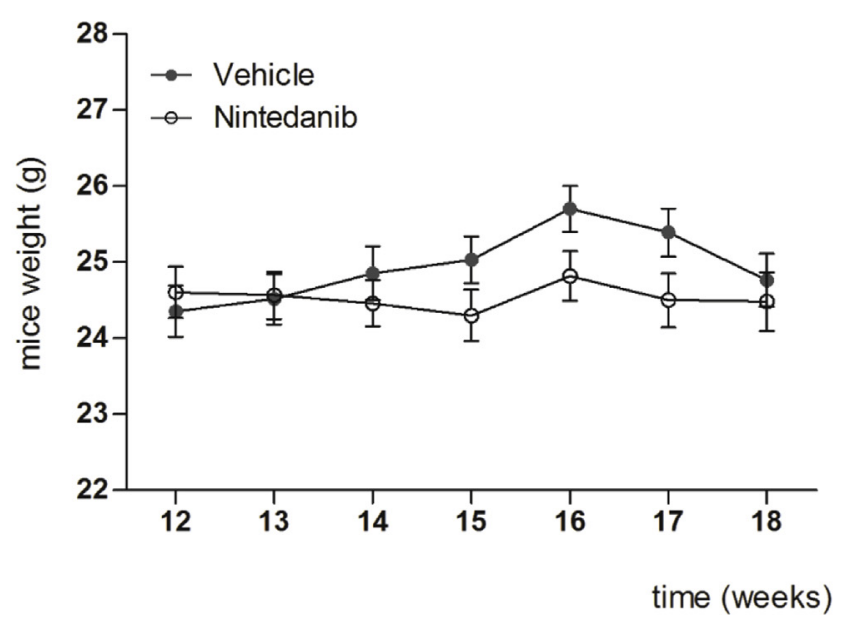

C
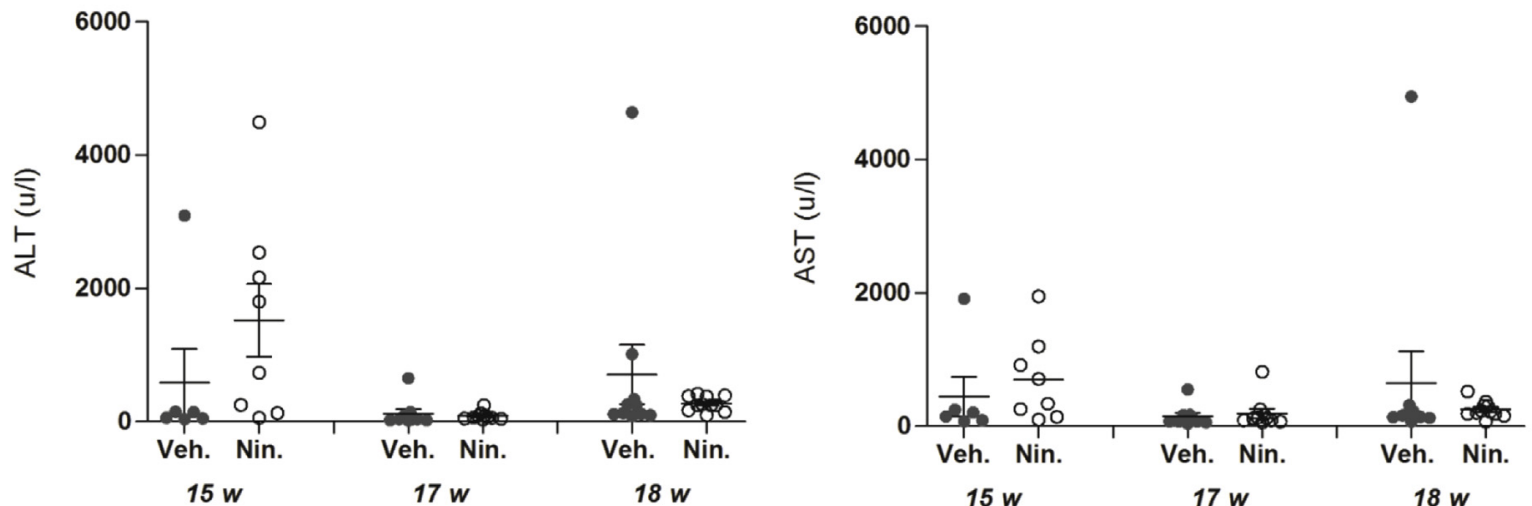

Supplementary Figure 7. Prolonged treatment with nintedanib does not induce significant toxicity in $\mathrm{DEN} / \mathrm{CCl}_{4}$-induced mouse models. $(A)$ Experimental design of the murine model of $\mathrm{DEN} / \mathrm{CCl}_{4}$-induced hepatocarcinogenesis in the context of liver fibrosis. $(B)$ Monitoring of body weight in all mice from each experimental group during the administration of nintedanib or vehicle at the longest time point, 18 weeks of age. $(C)$ Evaluation of the serum levels of alanine transaminase (ALT) and aspartate transaminase (AST) in each experimental group. Nin., nintedanib; Veh., vehicle. 
A

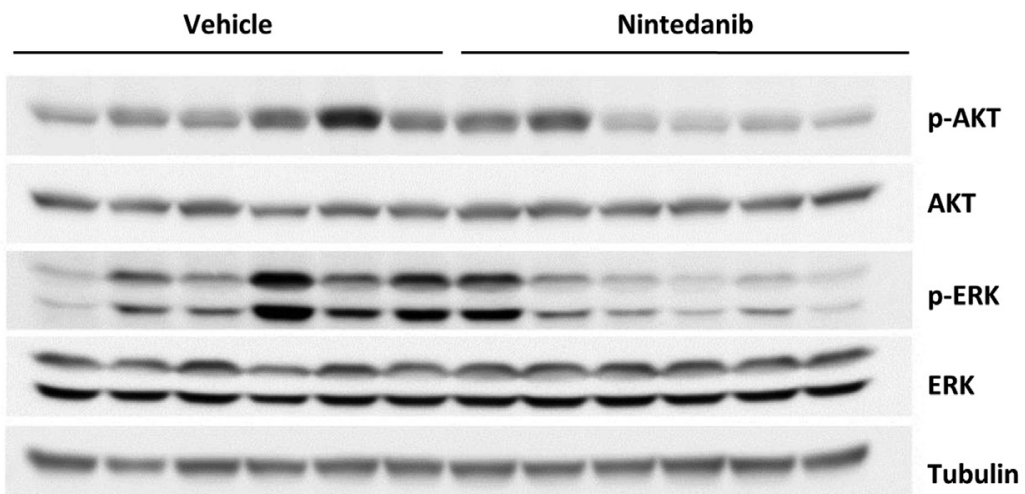

B
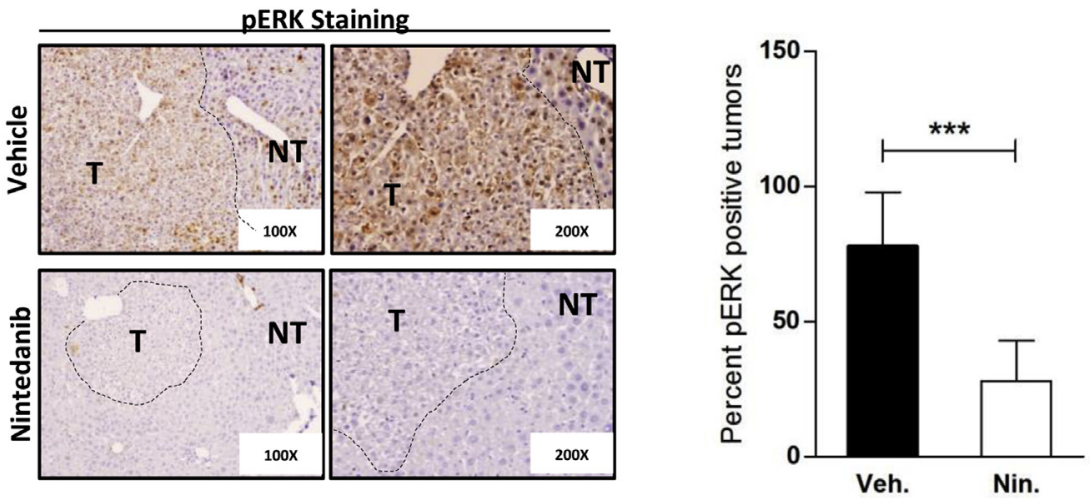

C

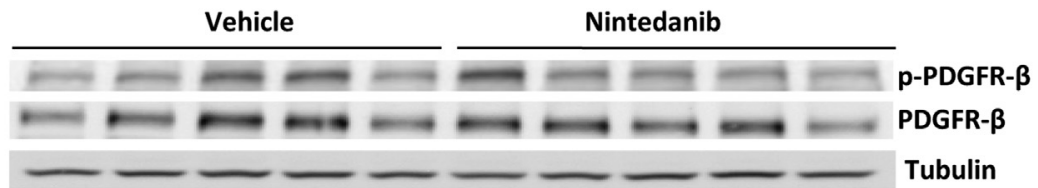

D
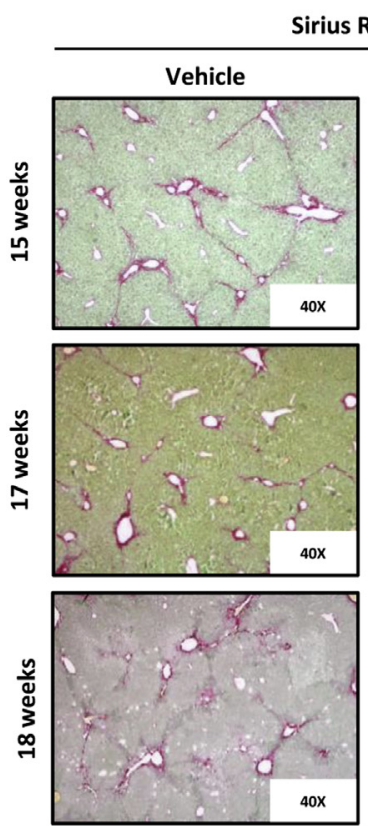

E
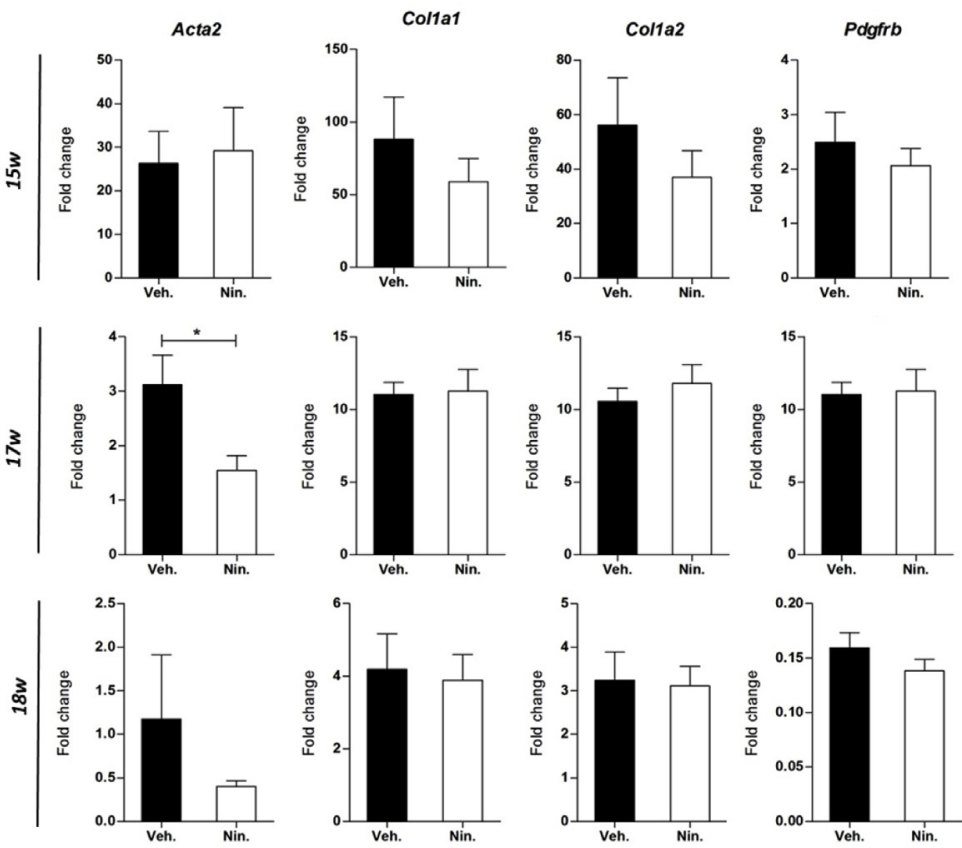
Supplementary Figure 8. Nintedanib inhibits the downstream mitogen-activated protein kinase pathway but has no effect on reverting $\mathrm{DEN} / \mathrm{CCl}_{4}$-induced platelet-derived growth factor signaling or hepatic fibrosis. $(A)$ Western-blot analysis of downstream mitogen-activated protein kinase (protein kinase B and ERK) signaling. (B) Representative images and quantification of pERK staining in 17-week-old mice treated with vehicle or nintedanib. In the vehicle arm, the tumors and adjacent nontumor tissue are positive with patchy nuclear and cytoplasmic staining, whereas in the nintedanib arm, both are negative. (C) Western blot analysis of the profibrogenic platelet-derived growth factor receptor signaling. $(D)$ Representative images of Sirius Red staining in mice treated with vehicle or nintedanib. Nintedanib did not exert any significant effect on liver fibrosis. ( $E$ ) Gene expression levels of profibrogenic marker genes by quantitative reverse-transcription polymerase chain reaction in livers of mice killed at 15, 17, and 18 weeks of age treated with vehicle or nintedanib. The GAPDH gene was used as a housekeeper for normalization. Significant statistical differences are defined as follows: ${ }^{\star} P<.05$ and ${ }^{\star \star \star} P<.001$. Nin., nintedanib; NT, nontumor; T, tumor; Veh., vehicle. 